\title{
Bichromatic Travelling Waves for Lattice Nagumo Equations*
}

\author{
Hermen Jan Hupkes ${ }^{\dagger}$, Leonardo Morelli ${ }^{\dagger}$, and Petr Stehlík ${ }^{\ddagger}$
}

\begin{abstract}
We discuss bichromatic (two-color) front solutions to the bistable Nagumo lattice differential equation. Such fronts connect the stable spatially homogeneous equilibria with spatially heterogeneous 2-periodic equilibria and hence are not monotonic like the standard monochromatic fronts. We provide explicit criteria that can determine whether or not these fronts are stationary and show that the bichromatic fronts can travel in parameter regimes where the monochromatic fronts are pinned. The presence of these bichromatic waves allows the two stable homogeneous equilibria to spread out through the spatial domain towards each other, buffered by a shrinking intermediate zone in which the periodic pattern is visible.
\end{abstract}

Key words. reaction-diffusion equation, lattice differential equation, travelling waves, nonlinear algebraic equations

AMS subject classifications. 34A33, 37L60, 39A12

DOI. $10.1137 / 18 \mathrm{M} 1189221$

1. Introduction. In this paper we consider the Nagumo lattice differential equation (LDE)

$$
\dot{u}_{j}(t)=d\left[u_{j-1}(t)-2 u_{j}(t)+u_{j+1}(t)\right]+g\left(u_{j}(t) ; a\right),
$$

posed on the spatial lattice $j \in \mathbb{Z}$, with $t \in \mathbb{R}$. We assume $d>0$ and use the standard cubic bistable nonlinearity $g(u ; a)=u(1-u)(u-a)$ with $a \in(0,1)$. This LDE is well known as a prototypical model that describes the competition between two stable states $u=0$ and $u=1$ in a discrete spatial environment. A crucial role is reserved for so-called travelling front solutions, which have the form

$$
u_{j}(t)=\Phi(j-c t), \quad \Phi(-\infty)=0, \quad \Phi(+\infty)=1 .
$$

Such solutions are often referred to as invasion waves, as they provide a mechanism by which the energetically preferred state can invade the spatial domain.

Our work focuses on the case where $c=0$ holds for these primary invasion waves, indicating a delicate balance between the two competing states. In this case (1.1) can admit stable

\footnotetext{
* Received by the editors May 23, 2018; accepted for publication (in revised form) by A. Scheel March 12, 2019; published electronically May 21, 2019.

http://www.siam.org/journals/siads/18-2/M118922.html

Funding: The work of the first author was supported by the Netherlands Organization for Scientific Research (NWO) grant 639.032.612. The work of the second author was supported by the Netherlands Organization for Scientific Research (NWO) grant 613.001.304. The work of the third author was supported by project LO1506 of the Czech Ministry of Education, Youth and Sports under the program NPU I.

${ }^{\dagger}$ Mathematisch Instituut, Universiteit Leiden, 2300 RA Leiden, The Netherlands (hhupkes@math.leidenuniv.nl, I.morelli@math.leidenuniv.nl).

${ }^{\ddagger}$ Department of Mathematics and NTIS, Faculty of Applied Sciences, University of West Bohemia, Univerzitní 8, 30614 Plzeň, Czech Republic (pstehlik@kma.zcu.cz).
} 
spatially periodic rest-states. Numerical results indicate that these states can act as a buffer between regions of space where $u=0$ and $u=1$ dominate the dynamics. This buffer shrinks as these two stable states appear to move towards each other. This latter process is governed by secondary two-component invasion waves that we analyze in detail in this paper.

Nagumo PDE. The LDE (1.1) can be seen as the nearest-neighbor discretization of the Nagumo reaction-diffusion PDE [35]

$$
u_{t}=u_{x x}+g(u ; a), \quad x \in \mathbb{R},
$$

on a spatial grid with size $h=d^{-1 / 2}$. This PDE has been used as a highly simplified model for the spread of genetic traits [1] and the propagation of electrical signals through nerve fibers [3]. In higher space dimensions it also serves as a desingularization of the standard mean-curvature flow that is often used to describe the evolution of interfaces [18].

Fife and McLeod [21] used phase plane analysis to show that (1.3) admits a front solution for each $a \in[0,1]$. Such solutions have the form

$$
u(x, t)=\Phi(x-c t), \quad \Phi(-\infty)=0, \quad \Phi(+\infty)=1,
$$

for some smooth waveprofile $\Phi$ and wavespeed $c$ that has the same sign as $a-\frac{1}{2}$. These fronts hence connect the two stable spatially homogeneous equilibria $u(x, t) \equiv 0$ and $u(x, t) \equiv 1$.

Exploiting the comparison principle, Fife and McLeod were able to show that these front solutions have a surprisingly large basin of attraction. Indeed, any solution to (1.3) with an initial condition $u(x, 0)=u_{0}(x)$ that has $u_{0}(x) \approx 0$ for $x \ll-1$ and $u_{0}(x) \approx 1$ for $x \gg+1$ will converge to a shifted version of this front as $t \rightarrow \infty$.

These front solutions can be used as building blocks to capture the behavior of a more general class of solutions to (1.3). Consider, for example, the two-parameter family of functions

$$
u_{\mathrm{plt} ; \alpha_{0}, \alpha_{1}}(x, t)=\Phi\left(x-c t+\alpha_{0}\right)+\Phi\left(-x-c t+\alpha_{1}\right)-1,
$$

with $\alpha_{1} \geq \alpha_{0}$. Each of these functions can be interpreted as a shifted version of the front solution (1.4) that is reflected in a vertical line to form a plateau.

If $c<0$, then any initial configuration that has $u_{0}(x) \approx 0$ for $|x| \gg L$ and $u_{0}(x) \approx 1$ for $|x| \leq L$ will converge to a member of the family (1.5) as $t \rightarrow \infty$. This provides a mechanism by which compact regions where $u \sim 1$ can spread out to fill the entire domain.

On the other hand, when $c>0$, one can construct entire solutions that converge to an element of (1.5) as $t \rightarrow-\infty$ and tend to zero as $t \rightarrow+\infty$. These solutions are stable under small perturbations [44]. In particular, they can be viewed as a robust elimination process whereby compact regions that have $u \sim 1$ are annihilated by two incoming travelling fronts that collide as $t \rightarrow \infty$.

Nagumo LDE. For many physical phenomena such as crystal growth in materials [6], the formation of fractures in elastic bodies [39], and the motion of dislocations [10] and domain walls [14] through crystals, the discreteness and topology of the underlying spatial domain have a major impact on the dynamical behavior. It is hence important to develop mathematical modelling tools that can incorporate such structures effectively. Indeed, by now it is well known that discrete models can capture dynamical behavior that their continuous counterparts cannot. 
The LDE (1.1) has served as a prototypical system in which such effects can be explored. It arises as a highly simplified model for the propagation of action potentials through nerve fibers that have regularly spaced gaps in their myelin coating [3]. Two-dimensional versions have been used to describe phase transitions in Ising models [2], to analyze predator-prey interactions [38], and to develop pattern recognition algorithms in image processing [12, 13]. Recently, an interest has also arisen in Nagumo equations posed on graphs [40], motivated by the network structure present in many biological systems [36].

Many authors have studied the LDE (1.1), focusing primarily on the richness of the set of equilibria [31] and the existence of travelling and standing front solutions [33, 45]. Such solutions have the form (1.2), which leads naturally to the waveprofile equation

$$
-c \Phi^{\prime}(\xi)=d[\Phi(\xi-1)-2 \Phi(\xi)+\Phi(\xi+1)]+g(\Phi(\xi) ; a) .
$$

Since the behavior of every lattice point is governed by the same profile $\Phi$, we refer to these front solutions as monochromatic waves in this paper (in order to distinguish them from the bichromatic waves we discuss in what follows). The seminal results by Mallet-Paret [33] show that for each $a \in[0,1]$ and $d>0$ there exists a unique $c=c_{\mathrm{mc}}(a, d)$ for which such monochromatic $(\mathrm{mc})$ solutions exist.

Pinning. Upon fixing $a \in(0,1) \backslash\left\{\frac{1}{2}\right\}$, Zinner [45] established that $c_{\mathrm{mc}}(a, d) \neq 0$ for $d \gg 1$, while Keener [30] showed that $c_{\mathrm{mc}}(a, d)=0$ for $0<d \ll 1$. Upon fixing $d>0$, Mallet-Paret established [33] that $c_{\mathrm{mc}}(a, d) \neq 0$ for $a \approx 1$ and $a \approx 0$. In addition, again for fixed $d>0$, the results in $[24,34]$ strongly suggest that there exists $\delta>0$ so that $c_{\mathrm{mc}}(a, d)=0$ whenever $\left|a-\frac{1}{2}\right| \leq \delta$; see Figure 1 .

This last phenomenon is called pinning and distinguishes the LDE (1.1) from the PDE (1.3). It is a direct consequence of the fact that we have broken the translational invariance of space. Indeed, (1.6) becomes singular in the limit $c \rightarrow 0$, and the corresponding waveprofiles indeed typically lose their smoothness. Many results suggest that this phenomenon is generic for discrete systems $[2,7,16,17,29]$. However, by carefully tuning the nonlinearity $g$ it is possible to design systems for which this pinning is absent $[15,26]$. Understanding the pinning phenomenon is an important and challenging mathematical problem that also has practical ramifications.

Periodicity. In this paper we study waves that connect spatially homogeneous stationary solutions of (1.1) with spatially heterogeneous 2-periodic stationary solutions. It is well known that many physical systems exhibit spatially periodic features [22, 23, 37]. Examples that also feature spatial discreteness include the presence of twinning microstructures in shape memory alloys [4] and the formation of domain-wall microstructures in dielectric crystals [41].

In many cases the underlying periodicity comes from the spatial system itself. For example, in $[19,20,25]$ the authors consider chains of alternating masses connected by identical springs (and vice versa). The dynamical behavior of such systems can be easily modelled by LDEs with periodic coefficients. In certain limiting cases the authors were able to construct so-called nanopterons, which are multicomponent wave solutions that have low-amplitude oscillations in their tails.

However, periodic patterns also arise naturally as solutions to spatially homogeneous discrete systems. Indeed, we shall see in section 3 that the LDE (1.1) with $d>0$ admits many 


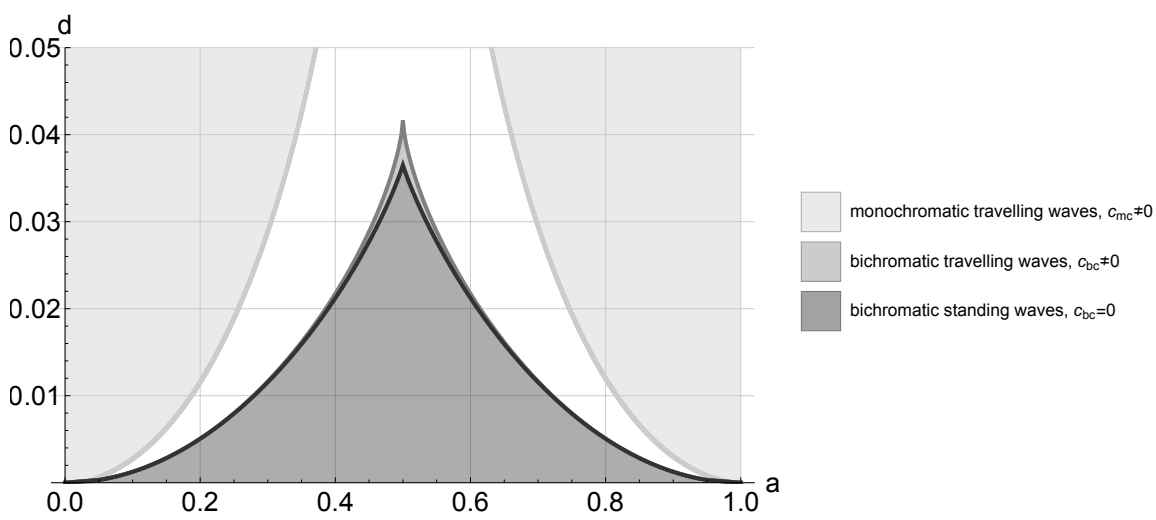

Figure 1. Existence regions for monochromatic and bichromatic wave solutions to (1.1).

periodic equilibria. In addition, the results in [31] explore the periodicity and chaos present in the set of equilibria to homogeneous LDEs with simplified nonlinearities.

It is also possible to introduce a natural periodicity into the structure of (1.1) by taking $d<0$. This can be seen by introducing new variables $v_{j}=(-1)^{j} u_{j}$, which restores the applicability of the comparison principle. This choice essentially decomposes the lattice sites $\mathbb{Z}$ into two groups, $\mathbb{Z}_{\text {odd }}$ and $\mathbb{Z}_{\text {even }}$, that each have their own characteristic behavior.

Such antidiffusion models have been used to describe phase transitions for grids of particles that have viscoelastic interactions $[8,9,42]$. In [5] this problem has been analyzed in considerable detail. The authors show that the resulting two-component system admits coexisting patterns that can be both monostable and bistable in nature. Similar results with piecewise linear nonlinearities but more general couplings between neighbors can be found in [43].

Bichromatic waves. In this paper we are interested in the parameter region where $c_{\mathrm{mc}}(a, d)=$ 0 . In a subset of this region it is possible to show that (1.1) has spatially heterogeneous stable equilibria. We focus on the simplest case and consider so-called bichromatic (two-color) equilibria, which are spatially periodic with period two. As such, they are closely connected to solutions of the Nagumo equation posed on a graph with two vertices. We set out to construct bichromatic front-solutions to (1.1), which can be seen as waves that connect the spatially homogeneous equilibrium $u \equiv 0$ with such a 2-periodic state. We emphasize that these differ from the traditional front-solutions (1.2) in the sense that the odd and even lattice sites each have their own waveprofile, as illustrated in Figure 2. Consequently, the bichromatic front-solutions are not monotone.

Our first main contribution is contained in section 3, where we give a detailed description of the set of parameters $(a, d)$ where such 2-periodic equilibria exist and where they are stable. In contrast to the setting encountered in [5], the relevant bifurcation curves cannot all be described explicitly. Besides a global result stating that the number of such equilibria decreases as $d$ is increased, we also obtain precise asymptotics that describe the boundaries near the three corners $(a, d) \in\{(0,0),(1 / 2,1 / 24),(1,0)\}$ in Figure 1.

As in [5], these preparations allow the existence of bichromatic fronts to be established in 

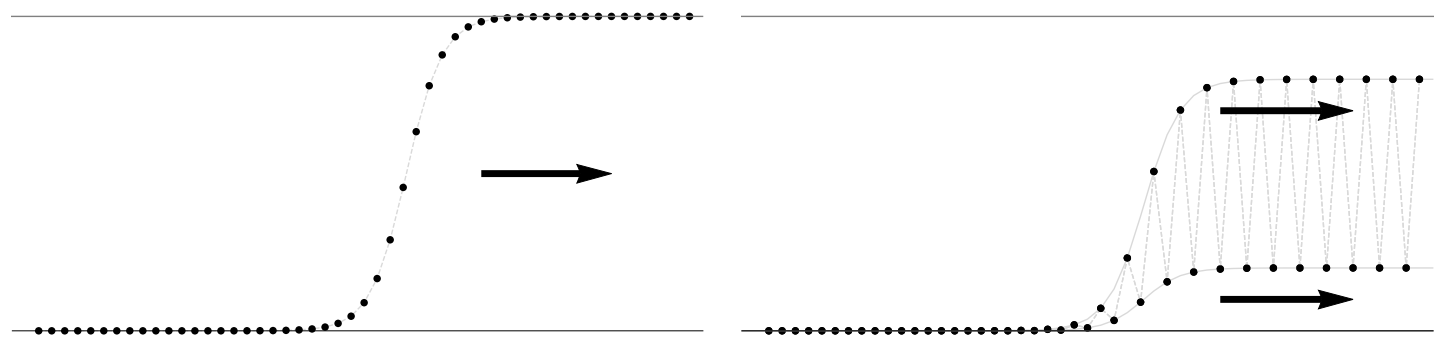

Figure 2. A monochromatic travelling wave of (1.1) (left panel) connects two spatially homogeneous stationary solutions. A bichromatic travelling wave of (1.1) (right panel) connects a spatially homogeneous stationary solution with a spatially heterogeneous one; see also M118922_02.mov [local/web 2.53MB].

a straightforward fashion. Indeed, one can apply the general theory developed by Chen, Guo, and $\mathrm{Wu}$ in [11] for discrete periodic systems that admit a comparison principle. These results imply that there exists a unique wavespeed $c_{\mathrm{bc}}(a, d)$ for which such bichromatic (bc) fronts exist. If $c_{\mathrm{bc}}(a, d) \neq 0$, the Fredholm theory developed in [28] together with the techniques from [27, section 3] can be used to show that these travelling fronts depend smoothly on $(a, d)$ and are nonlinearly stable.

However, these general results cannot distinguish between the cases $c_{\mathrm{bc}}(a, d)=0$ and $c_{\mathrm{bc}}(a, d) \neq 0$ where we have standing, respectively, travelling fronts. This should be contrasted to the situation for the PDE (1.3), where the sign of the wavespeed is given by the sign of a simple integral [21]. Indeed, there is a large set of parameters $(a, d)$ for which the discrete bichromatic fronts fail to travel, even though the analogous integral does not vanish.

Our second main contribution is that we provide explicit criteria in section 4 that can guarantee $c_{\mathrm{bc}}=0$ or $c_{\mathrm{bc}}>0$. Together these results cover most of the parameter region where bichromatic fronts exist. In any case, they provide a two-component generalization of the coercivity conditions introduced in [33], which ensure that $c_{\mathrm{mc}}(a, d) \neq 0$ for the boundary regions $a \approx 1$ and $a \approx 0$.

Our arguments to guarantee $c_{\mathrm{bc}}=0$ are closely related to the setup used by Keener [30] to establish that monochromatic waves are pinned for $0<d \ll 1$. In particular, for small values of $d$ one can neglect the diffusion term in section 1.1 and use properties of the cubic to show that the derivative of the waveprofile must change signs. This contradicts the fact that waveprofiles must be strictly monotonic if they travel.

On the other hand, in section 4 we develop an intuitive geometric construction involving reflections to describe a planar recurrence relation that standing bichromatic fronts must satisfy. This allows us to rule out the presence of such fronts when a scalar inequality is violated. This consequently implies the presence of travelling bichromatic fronts.

The parameter regimes where these two arguments apply both converge towards the corner points $(0,0)$ and $(1,0)$. Near these corners we need the delicate asymptotics described above to distinguish between the two cases.

Colliding fronts. One of the main reasons for our interest in these bichromatic fronts is that they present mechanisms via which the stable homogeneous states $u=0$ and $u=1$ can spread throughout the domain, even though the primary invasion waves are blocked from propagation. By using techniques similar to those in [44], we believe it should be possible 


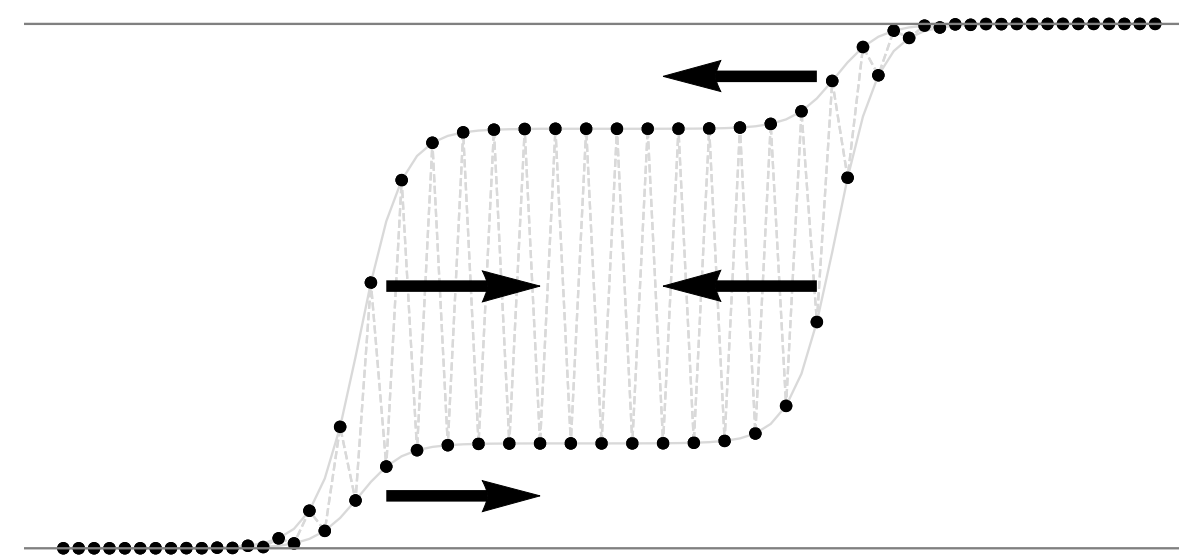

Figure 3. Colliding front of (1.1) consisting of a right-travelling bichromatic front connection between the homogeneous equilibrium $u \equiv 0$ and a 2-periodic intermediate state that collides with a left-travelling bichromatic connection between the 2-periodic state and the homogeneous equilibrium $u \equiv 1$. This is further illustrated in M118922_04.mov [local/web 926KB].

to construct entire solutions consisting of a right-travelling bichromatic front connection between the homogeneous equilibrium $u \equiv 0$ and a 2-periodic intermediate state that collides with a left-travelling bichromatic connection between a 2-periodic intermediate state and the homogeneous equilibrium $u \equiv 1$; see Figure 3. The resulting state after the collision is then a pinned monochromatic front that connects 0 with 1 . We have been able to numerically verify the existence of these solutions in the parameter regions predicted by the theory developed in this paper; see M118922_04.mov [local/web 926KB].

2. Main results. Our interest here is in the LDE

$$
\dot{x}_{j}(t)=d\left[x_{j-1}(t)-2 x_{j}(t)+x_{j+1}(t)\right]+g\left(x_{j}(t) ; a\right)
$$

posed on the one-dimensional lattice, i.e., $j \in \mathbb{Z}$. The bistable nonlinearity is explicitly given by

$$
g(u ; a)=u(1-u)(u-a),
$$

with $a \in(0,1)$. Our results concern so-called bichromatic (two-color) travelling wave solutions to the LDE (2.1). Such solutions can be written in the form

$$
x_{j}(t)= \begin{cases}\Phi_{u}(j-c t) & \text { if } j \text { is even, } \\ \Phi_{v}(j-c t) & \text { if } j \text { is odd, }\end{cases}
$$

for some wavespeed $c \in \mathbb{R}$ and $\mathbb{R}^{2}$-valued waveprofile

$$
\Phi=\left(\Phi_{u}, \Phi_{v}\right): \mathbb{R} \rightarrow \mathbb{R}^{2} .
$$


Substituting this Ansatz into (2.1), we obtain the travelling wave system

$$
\begin{aligned}
& -c \Phi_{u}^{\prime}(\xi)=d\left[\Phi_{v}(\xi-1)-2 \Phi_{u}(\xi)+\Phi_{v}(\xi+1)\right]+g\left(\Phi_{u}(\xi) ; a\right), \\
& -c \Phi_{v}^{\prime}(\xi)=d\left[\Phi_{u}(\xi-1)-2 \Phi_{v}(\xi)+\Phi_{u}(\xi+1)\right]+g\left(\Phi_{v}(\xi) ; a\right) .
\end{aligned}
$$

Upon introducing the functions

$$
G(u, v ; a, d)=\left(\begin{array}{c}
G_{1}(u, v ; a, d) \\
G_{2}(u, v ; a, d)
\end{array}\right)=\left(\begin{array}{c}
2 d(v-u)+g(u ; a) \\
2 d(u-v)+g(v ; a)
\end{array}\right),
$$

we see that any stationary solution

$$
\left(\Phi_{u}, \Phi_{v}\right)(\xi)=(\bar{u}, \bar{v})
$$

to (2.5) must satisfy the nonlinear algebraic equation

$$
G(\bar{u}, \bar{v} ; a, d)=0 .
$$

The full bifurcation diagram for this equation is described in section 3. For our purposes here, however, it suffices to summarize a subset of the conclusions from this analysis, which we do in our first result below. In particular, there exists a region $\Omega_{\mathrm{bc}}$ in the $(a, d)$-plane for which the spatially homogeneous system $(\dot{u}, \dot{v})=G(u, v ; a, d)$ has a stable equilibrium $\left(\bar{u}_{\mathrm{bc}}(a, d), \bar{v}_{\mathrm{bc}}(a, d)\right)$ that can be interpreted as a bichromatic equilibrium state for the LDE (2.1).

Proposition 2.1 (see section 3). There exists a continuous curve $d_{\mathrm{bc}}:[0,1] \rightarrow\left[0, \frac{1}{24}\right]$ with $d_{\mathrm{bc}}\left(\frac{1}{2}\right)=\frac{1}{24}$ and $d_{\mathrm{bc}}(1-a)=d_{\mathrm{bc}}(a)$ so that for every $0 \leq d<d_{\mathrm{bc}}$ and $0<a<1$ the system (2.8) has nine distinct equilibria $(\bar{u}, \bar{v}) \in[0,1]^{2}$. Upon writing

$$
\Omega_{\mathrm{bc}}=\left\{0<d<d_{\mathrm{bc}}(a) \text { and } 0<a<1\right\},
$$

there exist $C^{\infty}{ }_{- \text {smooth maps }}$

$$
\left(\bar{u}_{\mathrm{bc}}, \bar{v}_{\mathrm{bc}}\right): \Omega_{\mathrm{bc}} \rightarrow(0,1)^{2}
$$

with $\bar{u}_{\mathrm{bc}}<a<\bar{v}_{\mathrm{bc}}$ so that for every $(a, d) \in \Omega_{\mathrm{bc}}$ we have

$$
G\left(\bar{u}_{\mathrm{bc}}(a, d), \bar{v}_{\mathrm{bc}}(a, d) ; a, d\right)=0
$$

together with

$$
\operatorname{det} D_{1,2} G\left(\bar{u}_{\mathrm{bc}}(a, d), \bar{v}_{\mathrm{bc}}(a, d) ; a, d\right)>0, \quad \operatorname{Tr} D_{1,2} G\left(\bar{u}_{\mathrm{bc}}(a, d), \bar{v}_{\mathrm{bc}}(a, d) ; a, d\right)<0 .
$$

We note that the statements (2.11)-(2.12) are also valid upon replacing the bichromatic reststate $\left(\bar{u}_{\mathrm{bc}}, \bar{v}_{\mathrm{bc}}\right)$ by the monochromatic equilibria $(0,0)$ and $(1,1)$. We will be interested in waves that connect these monochromatic and bichromatic equilibria together. More precisely, we set out to find solutions to (2.5) that satisfy either the "lower" boundary conditions

$$
\lim _{\xi \rightarrow-\infty} \Phi(\xi)=(0,0), \quad \lim _{\xi \rightarrow+\infty} \Phi(\xi)=\left(\bar{u}_{\mathrm{bc}}, \bar{v}_{\mathrm{bc}}\right),
$$


or the "upper" boundary conditions

$$
\lim _{\xi \rightarrow-\infty} \Phi(\xi)=\left(\bar{u}_{\mathrm{bc}}, \bar{v}_{\mathrm{bc}}\right), \quad \lim _{\xi \rightarrow+\infty} \Phi(\xi)=(1,1) .
$$

Examples of such solutions can be found in M118922_02.mov [local/web 2.53MB], respectively, M118922_03.mov [local/web 723KB].

The result below summarizes several key facts concerning the existence and uniqueness of such waves. It introduces subregions of $\Omega_{\mathrm{bc}}$ denoted by $\mathcal{T}_{\text {low }}$ and $\mathcal{T}_{\text {up }}$ where the bichromatic travelling waves (2.3) exist with nonzero speeds $c_{\text {low }}>0$ and $c_{\text {up }}<0$; see Figure 4 . With the exception of the inequalities $c_{\text {low }} \geq 0$ and $c_{\text {up }} \leq 0$, these properties follow directly from the theory developed in $[11,28]$.

Theorem 2.2 (see section 4.1). There exist continuous maps

$$
c_{\text {low }}: \Omega_{\mathrm{bc}} \rightarrow[0, \infty), \quad c_{\mathrm{up}}: \Omega_{\mathrm{bc}} \rightarrow(-\infty, 0]
$$

that satisfy the following properties.

(i) Upon introducing the open sets

$$
\begin{aligned}
& \mathcal{T}_{\text {low }}=\left\{(a, d) \in \Omega_{\mathrm{bc}}: c_{\text {low }}>0\right\}, \\
& \mathcal{T}_{\text {up }}=\left\{(a, d) \in \Omega_{\mathrm{bc}}: c_{\mathrm{up}}<0\right\},
\end{aligned}
$$

the functions $c_{\text {low }}$ and $c_{\mathrm{up}}$ are $C^{\infty}$-smooth on $\mathcal{T}_{\text {low }}$, respectively, $\mathcal{T}_{\text {up }}$.

(ii) There exist $C^{\infty}{ }_{-}$smooth functions

$$
\Phi_{\text {low }}: \mathcal{T}_{\text {low }} \rightarrow W^{1 ; \infty}\left(\mathbb{R} ; \mathbb{R}^{2}\right), \quad \Phi_{\text {up }}: \mathcal{T}_{\text {up }} \rightarrow W^{1 ; \infty}\left(\mathbb{R} ; \mathbb{R}^{2}\right),
$$

such that for any $\# \in\{$ low, up $\}$ and any $(a, d) \in \mathcal{T}_{\#}$, the pair

$$
(c, \Phi)=\left(c_{\#}(a, d), \Phi_{\#}(a, d)\right)
$$

satisfies (2.5) together with the boundary condition (2.13) if \# = low or (2.14) if $\#=$ up. In addition, we have the componentwise inequality $\Phi_{\#}^{\prime}>(0,0)$.

(iii) For any $\# \in\{$ low, up $\}$ and any $(a, d) \in \Omega_{\mathrm{bc}} \backslash \mathcal{T}_{\#}$, there exists a nondecreasing function $\Phi: \mathbb{R} \rightarrow \mathbb{R}^{2}$ that satisfies (2.5) with $c=0$ together with the boundary condition (2.13) if $\#=$ low or (2.14) if \# = up.

(iv) Pick $\# \in\{$ low, up $\}$ and $(a, d) \in \Omega_{\mathrm{bc}}$, and consider any $c \neq 0$ together with a function $\Phi \in W^{1, \infty}\left(\mathbb{R} ; \mathbb{R}^{2}\right)$ that satisfies (2.5) together with the boundary condition (2.13) if $\#=$ low or (2.14) if \# = up. Then we must have $c=c_{\#}(a, d)$ and $\Phi=\Phi_{\#}(a, d)(\cdot-\vartheta)$ for some $\vartheta>0$. In particular, we have $(a, d) \in \mathcal{T}_{\#}$.

(v) Pick $\# \in\{$ low, up $\}$ and $(a, d) \in \Omega_{\mathrm{bc}}$, and consider any nondecreasing function $\Phi$ : $\mathbb{R} \rightarrow \mathbb{R}^{2}$ that satisfies (2.5) with $c=0$ together with the boundary condition (2.13) if $\#=$ low or (2.14) if \# = up. Then we must have $(a, d) \in \Omega_{\mathrm{bc}} \backslash \mathcal{T}_{\#}$.

We numerically determined the locations of the sets $\mathcal{T}_{\text {low }}$ and $\mathcal{T}_{\text {up }}$ in Figure 4 . In particular, we simulated (2.1) with an initial condition that consists of the stable 2-periodic pattern multiplied by a hyperbolic tangent. By checking if this solution converges to a travelling or 


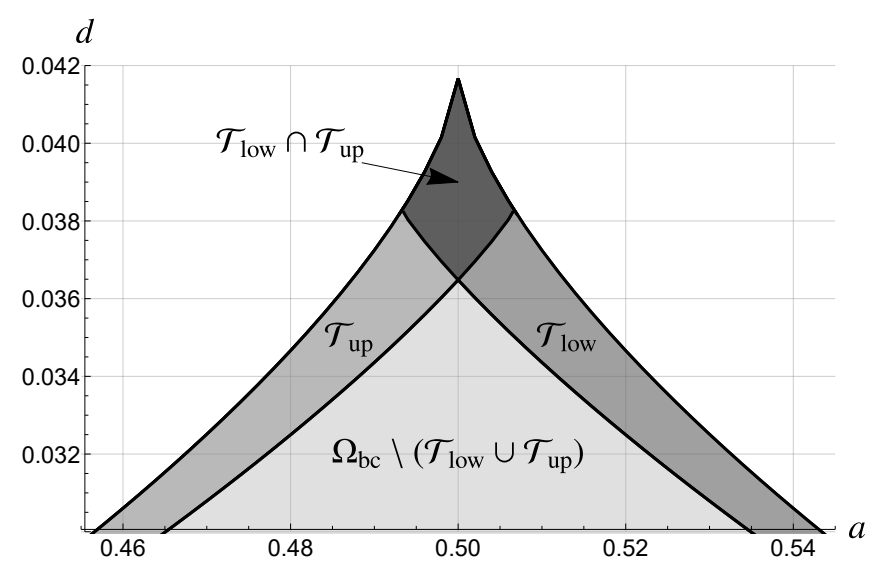

Figure 4. Numerical bounds for the parameter sets $\Omega_{b c}, \mathcal{T}_{\text {low }}$, and $\mathcal{T}_{\text {up }}$, introduced in Theorem 2.2 , in the neighborhood of the cusp $\left(\frac{1}{2}, \frac{1}{24}\right)$.

stationary wave, one can decide whether $(a, d) \in \mathcal{T}_{\text {low }}$. This procedure hinges on the crucial fact that the waves in item (ii) of Theorem 2.2 are nonlinearly stable under the dynamics of (1.1); see [11, Thm. 4].

The main contribution of the present paper is that we provide an explicit and verifiable inequality to rigorously confirm that the sets $\mathcal{T}_{\text {low }}$ and $\mathcal{T}_{\text {up }}$ touch the boundary curve $d=$ $d_{\mathrm{bc}}(a)$. In order to formulate this inequality, we first extend the bichromatic equilibria (2.10) to this boundary by writing

$$
\left(\bar{u}_{\mathrm{bc}}, \bar{v}_{\mathrm{bc}}\right)\left(a, d_{\mathrm{bc}}(a)\right)=\lim _{d \uparrow d_{\mathrm{bc}}(a)}\left(\bar{u}_{\mathrm{bc}}, \bar{v}_{\mathrm{bc}}\right)(a, d) .
$$

Inspection of (2.8) readily shows that this pair (2.19) is a solution to the fixed-point problem

$$
(u, v)=\left(u_{a}(v), v_{a}(u)\right)
$$

in which we have introduced the functions

$$
u_{a}(v)=v-\frac{g(v ; a)}{2 d_{\mathrm{bc}}(a)}, \quad v_{a}(u)=u-\frac{g(u ; a)}{2 d_{\mathrm{bc}}(a)} .
$$

The two critical points of these cubic functions are given by

$$
\gamma_{ \pm}(a)=\frac{1}{3}\left[a+1 \pm \sqrt{a^{2}-a+1-6 d_{\mathrm{bc}}(a)}\right] .
$$

Our approach relies on a geometric construction that rules out the existence of solutions to the recurrence relation that standing waves must satisfy. Indeed, in section 4.2 we will recast (2.5) with $c=0$ as a discrete map that involves horizontal and vertical reflections through the curves $u=u_{a}(v)$ and $v=v_{a}(u)$. Due to the monotonicity of the waveprofiles, several key features of the stable manifold for the pair (2.19) can therefore be captured by reflecting the lines $u=\bar{u}\left(a, d_{\mathrm{bc}}(a)\right)$ and $v=\bar{v}\left(a, d_{\mathrm{bc}}(a)\right)$ through these curves. 
The resulting reflected functions can be written as

$$
u_{a, r}(v)=2 u_{a}(v)-\bar{u}_{\mathrm{bc}}\left(a, d_{\mathrm{bc}}(a)\right), \quad v_{a, r}(u)=2 v_{a}(u)-\bar{v}_{\mathrm{bc}}\left(a, d_{\mathrm{bc}}(a)\right) .
$$

For any $a \in(0,1)$, we now define the set of intersections

$$
\mathcal{I}_{a}=\left\{(u, v): u=u_{a}(v) \text { and } v=v_{a, r}(u)\right\}
$$

which are all mapped onto the horizontal line $v=\bar{v}\left(a, d_{\mathrm{bc}}(a)\right)$. Assuming that this set is nonempty, we pick the specific representative

$$
u_{*}(a)=\max \left\{u: \text { there exists }(u, v) \in \mathcal{I}_{a} \text { with } 0 \leq u \leq \bar{u}_{\mathrm{bc}}\left(a, d_{\mathrm{bc}}(a)\right) \text { and } 0 \leq v \leq \gamma_{-}(a)\right\},
$$

which allows us to define the scalar function

$$
\Gamma(a)=u_{a, r}\left(\gamma_{+}(a)\right)-u_{*}(a) .
$$

The full geometric interpretation of this definition will be clarified and illustrated in section 4.2. For now, we simply remark that it measures the difference between two critical points associated to the unstable manifold of $(0,0)$ and the stable manifold of the pair $(2.19)$. When $\Gamma(a)>0$ holds, the critical points are ordered in such a way that connections between these two manifolds are blocked. This forces the inequality $c \neq 0$, implying that $\mathcal{T}_{\text {low }}$ indeed touches the boundary curve $d=d_{\mathrm{bc}}(a)$.

We wish to emphasize that there is an essential difference between computing $\Gamma(a)$ and simulating the full LDE (2.1) to check whether $c \neq 0$. Indeed, the first problem only requires knowledge of the curves (2.21)-(2.23) and is hence two-dimensional. On the other hand, the second problem is infinite dimensional and therefore necessarily involves truncations.

We are now ready to formulate our final result, which provides analytical bounds for the parameter regions $\mathcal{T}_{\text {low }}$ and $\mathcal{T}_{\text {up }}$ where the bichromatic waves actually travel (i.e., where $c_{\text {low }}>$ 0 , respectively, $\left.c_{\mathrm{up}}<0\right)$. Both regions contain a neighborhood of the cusp $(a, d)=\left(\frac{1}{2}, \frac{1}{24}\right)$. In addition, the corners $(0,0)$ and $(1,0)$ are accumulation points for the sets $\mathcal{T}_{\text {up }}$, respectively, $\mathcal{T}_{\text {low }}$.

Theorem 2.3 (see section 4.3). The sets $\mathcal{T}_{\text {low }}$ and $\mathcal{T}_{\text {up }}$ satisfy the following properties.

(i) For each $(a, d) \in \mathcal{T}_{\text {up }}$ we have $d>\frac{1}{8} a^{2}$, while for each $(a, d) \in \mathcal{T}_{\text {low }}$ we have $d>$ $\frac{1}{8}(1-a)^{2}$.

(ii) If $(a, d) \in \mathcal{T}_{\text {low }}$, then also $\left(a^{\prime}, d\right) \in \mathcal{T}_{\text {low }}$ for all $\left(a^{\prime}, d\right) \in \Omega_{\mathrm{bc}}$ that have $a^{\prime} \geq a$. On the other hand, if $(a, d) \in \mathcal{T}_{\text {up }}$, then also $\left(a^{\prime}, d\right) \in \mathcal{T}_{\text {up }}$ for all $\left(a^{\prime}, d\right) \in \Omega_{\mathrm{bc}}$ that have $a^{\prime} \leq a$.

(iii) There exists $\epsilon>0$ so that we have the inclusions

$$
(a, d) \in \mathcal{T}_{\text {low }} \cap \mathcal{T}_{\text {up }}
$$

for all $(a, d) \in \Omega_{\mathrm{bc}}$ that have

$$
0<\left|a-\frac{1}{2}\right|+\left|d-\frac{1}{24}\right|<\epsilon .
$$


(iv) The expression (2.26) is well defined for all $0<a<1$. If $\Gamma\left(a_{*}\right)>0$ for some $0<a_{*}<1$, then there exists $\epsilon>0$ so that $(a, d) \in \mathcal{T}_{\text {low }}$ for all $(a, d) \in \Omega_{\mathrm{bc}}$ that have

$$
0<\left|a-a_{*}\right|+\left|d-d_{\mathrm{bc}}\left(a_{*}\right)\right|<\epsilon .
$$

(v) The inequality $\Gamma(a)>0$ holds whenever $1-a>0$ is sufficiently small. In particular, we have $(0,0) \in \overline{\mathcal{T}}_{\text {up }}$ and $(1,0) \in \overline{\mathcal{T}}_{\text {low }}$.

Using numerics, we have verified that $\Gamma(a)>0$ holds for $a \in[.498, .999]$; see section 4.3. Together with (ii) and (v) above, this strongly suggests that $\mathcal{T}_{\text {low }}$ is a connected set that extends towards the right boundary of $\Omega_{\mathrm{bc}}$. By symmetry, this is equivalent to the statement that $\mathcal{T}_{\text {up }}$ is a connected set that extends towards the left boundary of $\Omega_{\mathrm{bc}}$.

3. Bichromatic stationary solutions. In this section we uncover the structure of the solution set to $G(u, v ; a, d)=0$ as a function of the parameters $(a, d)$. Our first result shows that for $d \gg 1$ this equation only has the monochromatic roots $(0,0),(a, a)$, and $(1,1)$. The threshold $d_{+}(a)$ between this region and the region with five distinct roots can be explicitly computed. However, we only have qualitative and asymptotic results for the boundary $d_{-}(a)$ where the root-count increases to the maximal value of nine. In Figure 5 we compare these asymptotics to numerically computed values for $d_{-}(a)$.

We remark here that the monotonicity of the root-count with respect to $d$ does not hold for general bistable nonlinearities $g$. Although the behavior for large and small values of $d$ is universal, additional bifurcation curves can (and do) occur between $d_{-}$and $d_{+}$. In light of this, it is not surprising that several of our results require us to use the precise formula for the cubic. Of course, the explicit expansions discussed above for the curve $d_{-}(a)$ also depend on the specific form of $g(u ; a)$, but these can readily be recomputed if the nonlinearity is changed.

Proposition 3.1 (see section 3.3). There exist two continuous functions

$$
d_{ \pm}:[0,1] \rightarrow[0, \infty)
$$

that satisfy the following properties.

(i) For any $0<a<1$ we have the explicit expression

$$
d_{+}(a)=\frac{g^{\prime}(a ; a)}{4}
$$

together with the identities

$$
d_{-}(a)=d_{-}(1-a), \quad d_{+}(a)=d_{+}(1-a)
$$

and the inequality $d_{-}(a)<d_{+}(a)$. In addition, we have

$$
d_{-}(0)=d_{+}(0)=d_{-}(1)=d_{+}(1)=0
$$

together with $d_{-}\left(\frac{1}{2}\right)=\frac{1}{24}$.

(ii) We have $d_{-} \in C^{\infty}\left(\left[0, \frac{1}{2}\right)\right) \cap C^{\infty}\left(\left(\frac{1}{2}, 1\right]\right)$. In addition, $d_{-}$is strictly increasing on $\left[0, \frac{1}{2}\right]$ and strictly decreasing on $\left[\frac{1}{2}, 1\right]$. 


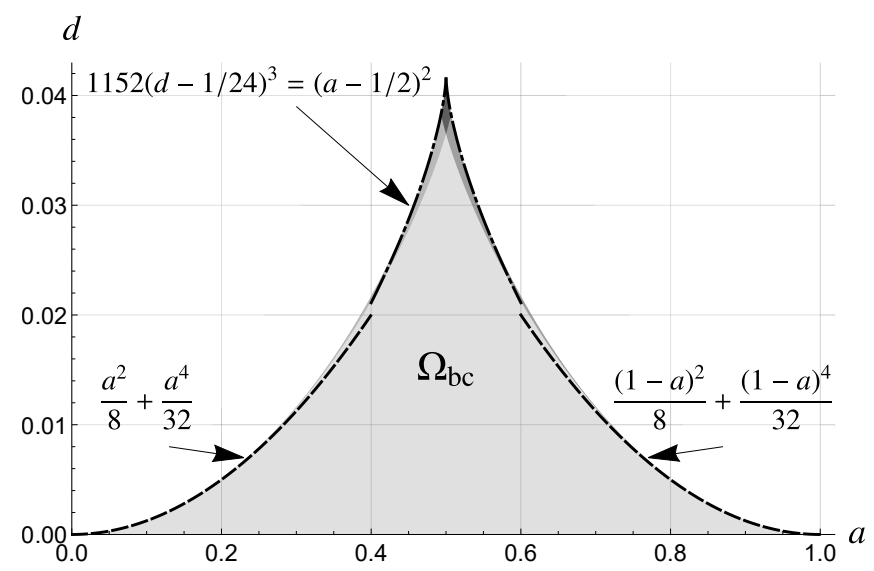

Figure 5. Comparison of the asymptotics for $d_{-}$described in Proposition 3.1(v) with the numerically computed border of the set $\Omega_{-}$.

(iii) Pick any $a \in(0,1)$. The equation $G(u, v ; a, d)=0$ has nine distinct roots for $0 \leq$ $d<d_{-}(a)$, five distinct roots for $d_{-}<d<d_{+}(a)$, and three distinct real roots for $d \geq d_{+}(a)$.

(iv) Pick any $a \in(0,1)$. The equation $G(u, v ; a, d)=0$ has seven distinct roots for $d=$ $d_{-}(a)$ if $a \neq \frac{1}{2}$ and five if $a=\frac{1}{2}$.

(v) We have the expansion $d_{-}(a)=\frac{1}{8} a^{2}+\frac{1}{32} a^{4}+O\left(a^{5}\right)$ for $a \downarrow 0$. In addition, writing $a_{-}:\left[0, \frac{1}{24}\right] \rightarrow\left[0, \frac{1}{2}\right]$ for the inverse function of $d_{-}$on $\left[0, \frac{1}{2}\right]$, we have the expansion

$$
a_{-}(d)=\frac{1}{2}-\sqrt{-1152\left(d-\frac{1}{24}\right)^{3}}+O\left(\left(d-\frac{1}{24}\right)^{2}\right)
$$

as $d \uparrow \frac{1}{24}$.

In order to break the symmetry caused by the swap $u \leftrightarrow v$, we set out to describe the roots of $G(u, v ; a, d)=0$ that have $v>u$. To this end, we introduce two regions,

$$
\begin{aligned}
& \Omega_{-}=\left\{(a, d): 0<a<1 \text { and } 0<d<d_{-}(a)\right\}, \\
& \Omega_{+}=\left\{(a, d): 0<a<1 \text { and } d_{-}(a)<d<d_{+}(a)\right\},
\end{aligned}
$$

that are studied separately in the two results below. In $\Omega_{-}$there are three such bichromatic equilibria with $v>u$. These equilibria can be ordered, and the middle one is the only stable one. Two (or three) of these equilibria collide at $d=d_{-}(a)$ in a saddle node (or pitchfork) bifurcation, leaving a single unstable bichromatic equilibrium in $\Omega_{+}$. This equilibrium in turn collides with its swapped counterpart and the monochromatic equilibrium $(a, a)$ on the boundary $d_{+}(a)$. These processes are illustrated in Figure 6 . In particular, we see that $\Omega_{-}$ coincides with the set $\Omega_{\mathrm{bc}}$ introduced in section 2; cf. Figures 4 and 5 .

Proposition 3.2 (see section 3.3). There exist continuous functions

$$
\left(\bar{u}_{A}, \bar{v}_{A}\right): \bar{\Omega}_{-} \rightarrow[0,1]^{2}, \quad\left(\bar{u}_{B}, \bar{v}_{B}\right): \bar{\Omega}_{-} \rightarrow[0,1]^{2}, \quad\left(\bar{u}_{C}, \bar{v}_{C}\right): \bar{\Omega}_{-} \rightarrow[0,1]^{2}
$$

that satisfy the following properties. 

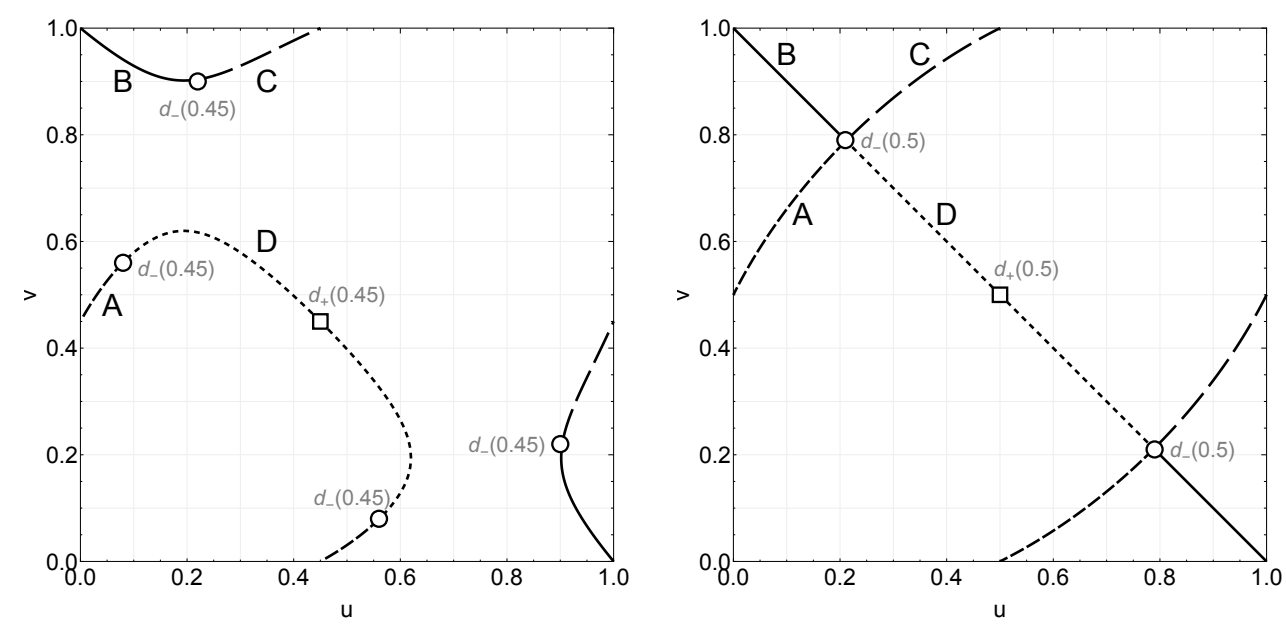

Figure 6. Illustration of the functions $\left(\bar{u}_{A}, \bar{v}_{A}\right),\left(\bar{u}_{B}, \bar{v}_{B}\right),\left(\bar{u}_{C}, \bar{v}_{C}\right)$ from Proposition 3.2 and the function $\left(\bar{u}_{D}, \bar{v}_{D}\right)$ from Proposition 3.3 for $a=.45$ (left panel) and $a=.5$ (right panel). The bifurcations occurring at $d_{+}(a)$ and $d_{-}(a)$ are indicated by squares and circles; see Proposition 3.1.

(i) Pick any $(a, d) \in \bar{\Omega}_{-}$. Then we have

$$
G\left(\bar{u}_{\#}(a, d), \bar{v}_{\#}(a, d) ; a, d\right)=0
$$

for all $\# \in\{A, B, C\}$. If also $(a, d) \in \Omega_{-}$, then the matrix

$$
D_{1,2} G\left(\bar{u}_{\#}(a, d), \bar{v}_{\#}(a, d) ; a, d\right)
$$

has two strictly negative eigenvalues if $\#=B$ or one strictly positive and one strictly negative eigenvalue if $\# \in\{A, C\}$.

(ii) For any $0 \leq a \leq 1$ we have the identities

$$
\left(\bar{u}_{A}, \bar{v}_{A}\right)(a, 0)=(0, a), \quad\left(\bar{u}_{B}, \bar{v}_{B}\right)(a, 0)=(0,1), \quad\left(\bar{u}_{C}, \bar{v}_{C}\right)(a, 0)=(a, 1) .
$$

(iii) For any $(a, d) \in \Omega_{-}$we have the ordering

$$
0<\bar{u}_{A}(a, d)<\bar{u}_{B}(a, d)<\bar{u}_{C}(a, d)<a<\bar{v}_{A}(a, d)<\bar{v}_{B}(a, d)<\bar{v}_{C}(a, d) .
$$

(iv) For any $a \in\left[0, \frac{1}{2}\right]$ we have

$$
\left(\bar{u}_{B}, \bar{v}_{B}\right)\left(a, d_{-}(a)\right)=\left(\bar{u}_{C}, \bar{v}_{C}\right)\left(a, d_{-}(a)\right),
$$

while for any $a \in\left[\frac{1}{2}, 1\right]$ we have

$$
\left(\bar{u}_{A}, \bar{v}_{A}\right)\left(a, d_{-}(a)\right)=\left(\bar{u}_{B}, \bar{v}_{B}\right)\left(a, d_{-}(a)\right) .
$$

Proposition 3.3 (see section 3.3). There exist continuous functions

$$
\left(\bar{u}_{D}, \bar{v}_{D}\right): \bar{\Omega}_{+} \rightarrow[0,1]^{2}
$$

that satisfy the following properties. 
(i) Pick any $(a, d) \in \bar{\Omega}_{+}$. Then we have

$$
G\left(\bar{u}_{D}(a, d), \bar{v}_{D}(a, d) ; a, d\right)=0 .
$$

If also $(a, d) \in \Omega_{+}$, then the matrix

$$
D_{1,2} G\left(\bar{u}_{D}(a, d), \bar{v}_{D}(a, d) ; a, d\right)
$$

has one strictly positive eigenvalue and one strictly negative eigenvalue.

(ii) For any $0 \leq a \leq 1$ we have the identity

$$
\left(\bar{u}_{D}, \bar{v}_{D}\right)\left(a, d_{+}(a)\right)=(a, a)
$$

(iii) For any $(a, d) \in \Omega_{+}$we have the ordering

$$
0<\bar{u}_{D}(a, d)<a<\bar{v}_{D}(a, d)<1
$$

(iv) For any $a \in\left[0, \frac{1}{2}\right]$ we have the identity

$$
\left(\bar{u}_{D}, \bar{v}_{D}\right)\left(a, d_{-}(a)\right)=\left(\bar{u}_{A}, \bar{v}_{A}\right)\left(a, d_{-}(a)\right),
$$

while for any $a \in\left[\frac{1}{2}, 1\right]$ we have

$$
\left(\bar{u}_{D}, \bar{v}_{D}\right)\left(a, d_{-}(a)\right)=\left(\bar{u}_{C}, \bar{v}_{C}\right)\left(a, d_{-}(a)\right)
$$

Corollary 3.4. For any $(a, d) \in \bar{\Omega}_{-}$, we have the identities

$$
\begin{aligned}
& \left(\bar{u}_{A}, \bar{v}_{A}\right)(a, d)=\left(1-\bar{v}_{C}, 1-\bar{u}_{C}\right)(1-a, d), \\
& \left(\bar{u}_{B}, \bar{v}_{B}\right)(a, d)=\left(1-\bar{v}_{B}, 1-\bar{u}_{B}\right)(1-a, d), \\
& \left(\bar{u}_{C}, \bar{v}_{C}\right)(a, d)=\left(1-\bar{v}_{A}, 1-\bar{u}_{A}\right)(1-a, d) .
\end{aligned}
$$

In addition, for any $(a, d) \in \bar{\Omega}_{+}$we have the identity

$$
\left(\bar{u}_{D}, \bar{v}_{D}\right)(a, d)=\left(1-\bar{v}_{D}, 1-\bar{u}_{D}\right)(1-a, d)
$$

Proof. The symmetry $g(1-u, 1-a)=-g(u, a)$ implies that

$$
G(1-u, 1-v ; 1-a, d)=-G(u, v ; a, d) .
$$

In addition, we have $G(u, v ; a, d)=0$ if and only if $G(v, u ; a, d)=0$. The statements hence follow from the ordering (3.11).

Our final result concerns the special case $a=\frac{1}{2}$, in which case it is possible to be more explicit. In particular, the bichromatic roots $\left(\bar{u}_{B}, \bar{v}_{B}\right)$ and $\left(\bar{u}_{D}, \bar{v}_{D}\right)$ lie on the line $u+v=1$ and collide precisely when $g^{\prime}\left(u ; \frac{1}{2}\right)=g^{\prime}\left(v ; \frac{1}{2}\right)=0$. 
Corollary 3.5. For any $0 \leq d \leq \frac{1}{24}$ we have

$$
\bar{u}_{B}\left(\frac{1}{2}, d\right)=1-\bar{v}_{B}\left(\frac{1}{2}, d\right)
$$

while for any $\frac{1}{24} \leq d \leq \frac{1}{16}=d_{+}(1 / 2)$ we have

$$
\bar{u}_{D}\left(\frac{1}{2}, d\right)=1-\bar{v}_{D}\left(\frac{1}{2}, d\right) .
$$

In addition, we have the identities

$$
\begin{aligned}
& \bar{u}_{A}\left(\frac{1}{2}, \frac{1}{24}\right)=\bar{u}_{B}\left(\frac{1}{2}, \frac{1}{24}\right)=\bar{u}_{C}\left(\frac{1}{2}, \frac{1}{24}\right)=\bar{u}_{D}\left(\frac{1}{2}, \frac{1}{24}\right)=\frac{1}{2}-\frac{1}{6} \sqrt{3}, \\
& \bar{v}_{A}\left(\frac{1}{2}, \frac{1}{24}\right)=\bar{v}_{B}\left(\frac{1}{2}, \frac{1}{24}\right)=\bar{v}_{C}\left(\frac{1}{2}, \frac{1}{24}\right)=\bar{u}_{D}\left(\frac{1}{2}, \frac{1}{24}\right)=\frac{1}{2}+\frac{1}{6} \sqrt{3} .
\end{aligned}
$$

3.1. Geometry of the cubic. Our strategy to establish Propositions 3.1-3.3 hinges upon geometric properties of the cubic $g(u ; a)$. As preparation, we introduce the notation

$$
u_{\text {infl }}(a)=\frac{1}{3}(a+1)
$$

together with

$$
u_{\text {min }}(a)=u_{\text {infl }}(a)-\frac{1}{3} \sqrt{1-a(1-a)}, \quad u_{\max }(a)=u_{\text {infl }}(a)+\frac{1}{3} \sqrt{1-a(1-a)}
$$

and note that

$$
g^{\prime}\left(u_{\min }(a) ; a\right)=0, \quad g^{\prime \prime}\left(u_{\mathrm{infl}}(a) ; a\right)=0, \quad g^{\prime}\left(u_{\max }(a) ; a\right)=0 .
$$

In addition, we note that $g^{\prime \prime}(u ; a)>0$ for $u<u_{\text {infl }}(a)$ and $g^{\prime \prime}(u ; a)<0$ for $u>u_{\text {infl }}(a)$. When $0<a<\frac{1}{2}$, we have the ordering

$$
0<u_{\min }(a)<a<u_{\text {infl }}(a)<u_{\max }(a)<1 .
$$

Recalling the definition (2.6), one readily sees that

$$
G_{1}(u, v ; a, d)+G_{2}(u, v ; a, d)=g(u ; a)+g(v ; a) .
$$

In order to exploit the fact that this identity does not depend on $d$, we first use basic properties of the cubic to parametrize solutions to $g(u ; a)+g(v ; a)=0$. Restricting ourselves to $a \in\left[0, \frac{1}{2}\right]$, it is possible to construct two solution curves $v_{ \pm}(u)$ that are defined for $u \in[0, a]$; see Figure 7 .

Lemma 3.6. Fix $0<a<\frac{1}{2}$. Then there are two constants

$$
a<v_{-; \max }<v_{+; \min }<1
$$

together with two $C^{\infty}$-smooth functions

$$
v_{-}:[0, a] \rightarrow\left[a, v_{-; \max }\right], \quad v_{+}:[0, a] \rightarrow\left[v_{+; \min }, 1\right]
$$

that satisfy the following properties. 

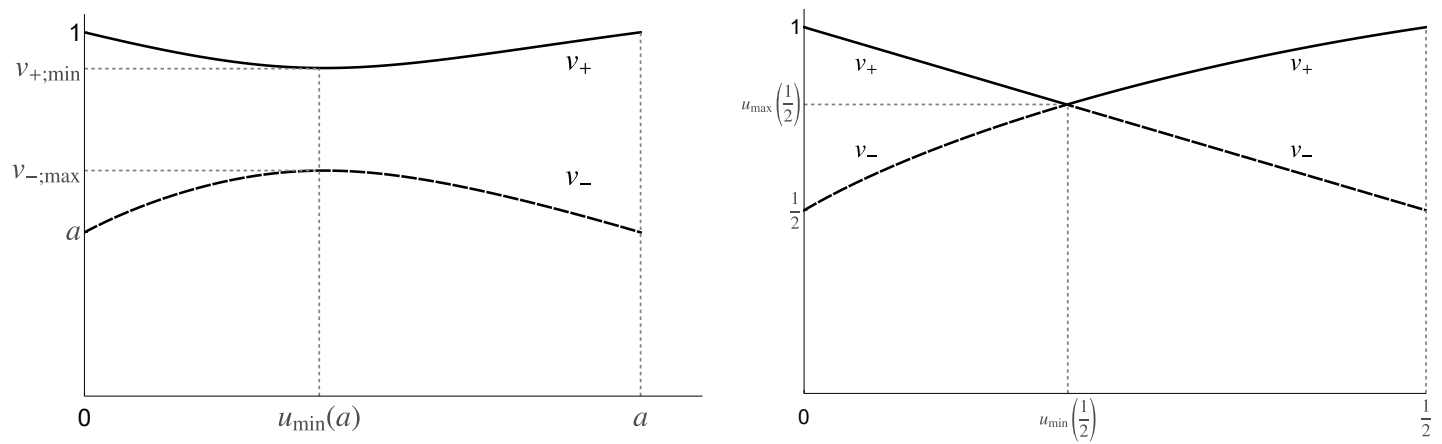

Figure 7. The functions $v_{ \pm}$defined in Lemmas 3.6-3.7 for $a=0.45$ (left) and $a=0.5$ (right). In the latter case the derivatives have a discontinuity at $u_{\min }\left(\frac{1}{2}\right)$.

(i) We have $g(u ; a)=-g\left(v_{-}(u) ; a\right)=-g\left(v_{+}(u) ; a\right)$ for all $0 \leq u \leq a$.

(ii) If $g(u ; a)=-g(v ; a)$ for some pair $u \in(0, a)$ and $v \in[0,1]$, then $v=v_{-}(u)$ or $v=v_{+}(u)$

(iii) We have $v_{-}(0)=v_{-}(a)=a$ and $v_{+}(0)=v_{+}(a)=1$.

(iv) We have the identities

$$
v_{ \pm}^{\prime}(u)=-\left[g^{\prime}\left(v_{ \pm}(u) ; a\right)\right]^{-1} g^{\prime}(u ; a)
$$

for all $0 \leq u \leq a$.

(v) We have $v_{-}^{\prime}(u)>-1$ for all $0 \leq u<a$, together with $v_{-}^{\prime}(a)=-1$.

Proof. Since $0<a<\frac{1}{2}$, we have $g\left(u_{\max }(a) ; a\right)>-g\left(u_{\min }(a) ; a\right)$, which implies that $v_{ \pm}(u) \neq u_{\max }(a)$. Properties (i)-(iv) hence follow immediately from the implicit function theorem.

To obtain (v), we take $u_{\min }(a)<u<a$ and recall $a<v_{-}(u)<u_{\max }$. If $v_{-}(u) \leq u_{\text {infl }}(a)$, then clearly $g^{\prime}\left(v_{-}(u) ; a\right)>g^{\prime}(u ; a)>0$, as desired. In order to handle the remaining case $v_{-}(u)>u_{\text {infl }}(a)$, we introduce the reflection $u_{\text {refl }}=2 u_{\text {infl }}(a)-u$. Exploiting the point symmetry of the graph of $g(\cdot ; a)$ around its inflection point $\left(u_{\mathrm{infl}}(a), g\left(u_{\mathrm{inf}}(a) ; a\right)\right)$, the inequality $g\left(u_{\text {infl }}(a) ; a\right)>0$ implies that

$$
u_{\text {infl }}(a)<v_{-}(u)<u_{\text {refl }}
$$

Since $g^{\prime \prime}(\tilde{u} ; a)<0$ for $\tilde{u}>u_{\text {infl }}(a)$, we obtain

$$
g^{\prime}\left(v_{-}(u) ; a\right)>g^{\prime}\left(u_{\text {reff }} ; a\right)=g^{\prime}(u ; a)>0,
$$

which implies $v_{-}^{\prime}(u)>-1$.

Lemma 3.7. Fix $a=\frac{1}{2}$. Then there are two functions

$$
v_{-}:[0, a] \rightarrow\left[a, u_{\max }(a)\right], \quad v_{+}:[0, a] \rightarrow\left[u_{\max }(a), 1\right]
$$

that satisfy items (i)-(iii) from Lemma 3.6 together with the following additional properties. 
(i) We have

$$
v_{-}\left(u_{\min }(a)\right)=v_{+}\left(u_{\min }(a)\right)=u_{\max }(a) .
$$

(ii) For any $u \in[0, a] \backslash\left\{u_{\min }(a)\right\}$ we have the identities

$$
v_{ \pm}^{\prime}(u)=-\left[g^{\prime}\left(v_{ \pm}(u) ; a\right)\right]^{-1} g^{\prime}(u ; a) .
$$

(iii) We have $v_{-}(u)=1-u$ for all $u_{\min }(a) \leq u \leq 1$, while $v_{+}(u)=1-u$ for all $0 \leq u \leq$ $u_{\min }(a)$.

(iv) We have the limits

$$
\lim _{u \uparrow u_{\min }(a)} v_{-}^{\prime}(u)=\lim _{u \downarrow u_{\min }(a)} v_{+}^{\prime}(u)=1
$$

together with

$$
\lim _{u \uparrow u_{\min }(a)} v_{-}^{\prime \prime}(u)=\lim _{u \downarrow u_{\min }(a)} v_{+}^{\prime \prime}(u)=-\frac{4}{3} \sqrt{3}
$$

Proof. Items (i) and (iii) follow directly from the symmetry of $g(\cdot ; a)$, while (ii) follows from the implicit function theorem. To obtain (iv), we first compute

$$
g\left(u_{\min }+u ; a\right)=g\left(u_{\min } ; a\right)+\frac{1}{2} \sqrt{3} u^{2}-u^{3}, \quad g\left(u_{\max }+v ; a\right)=-g\left(u_{\min } ; a\right)-\frac{1}{2} \sqrt{3} v^{2}-v^{3}
$$

In particular, the identity

$$
g\left(u_{\min }+u ; a\right)=-g\left(u_{\max }+v ; a\right)
$$

can be rewritten as

$$
v^{2}=u^{2}-\frac{2}{3} \sqrt{3}\left[u^{3}+v^{3}\right]
$$

which can be interpreted as a fixed point problem for $v^{2}$ upon assuming that $v$ and $u$ have the same sign. For small $|u|$ this problem has a solution that can be expanded as

$$
v^{2}=u^{2}-\frac{4}{3} \sqrt{3} u^{3}+O\left(u^{4}\right)
$$

which yields

$$
v=u-\frac{2}{3} \sqrt{3} u^{2}+O\left(u^{3}\right)
$$

The desired limits follow directly from this expansion. 
3.2. Tangencies. Let us now fix $a \in\left[0, \frac{1}{2}\right]$. In order to find solutions to $G(u, v ; a, d)=0$ with $d>0$, we introduce the function

$$
v_{d}(u)=u-\frac{g(u ; a)}{2 d}
$$

and note that the results above show that it suffices to find $u \in[0, a]$ for which one of the equations

$$
v_{ \pm}(u)=v_{d}(u)
$$

holds.

Our goal here is to show that nontransverse intersections of this type can only occur at local minima of $v_{ \pm}-v_{d}$. Together with the strict monotonicity

$$
\partial_{d} v_{d}(u)<0
$$

that holds for $u \in(0, a)$, this will allow us to obtain global results in section 3.3 .

For the moment, we note that

$$
v_{-}(a)=v_{d}(a)=a
$$

for every $d>0$. In addition, we may compute

$$
v_{d}^{\prime}(a)=1-\frac{g^{\prime}(a ; a)}{2 d},
$$

together with

$$
v_{-}^{\prime}(a)=-\left[g^{\prime}(a ; a)\right]^{-1} g^{\prime}(a ; a)=-1 .
$$

In particular, when $g^{\prime}(a)=4 d$, the intersection (3.50) is tangential. In what follows we show that in fact $v_{-}>v_{d}$ on $[0, a)$ for this critical value of $d$.

For intersections with $u \in(0, a)$ such explicit computations are significantly harder to carry out, which is why we pursue a more indirect approach here. As preparation, we compute

$$
v_{ \pm}^{\prime \prime}(u)=-\frac{g^{\prime \prime}(u)}{g^{\prime}\left(v_{ \pm}(u)\right)}-\frac{g^{\prime}(u)^{2}}{g^{\prime}\left(v_{ \pm}(u)\right)^{3}} g^{\prime \prime}\left(v_{ \pm}(u)\right)
$$

together with

$$
v_{d}^{\prime \prime}(u)=-\frac{g^{\prime \prime}(u)}{2 d} .
$$

In addition, for any $\kappa \leq g^{\prime}\left(u_{\text {infl }}(a) ; a\right)=\frac{1}{3}\left(a^{2}-a+1\right)$, we introduce the expressions

$$
\begin{aligned}
& u_{l}(\kappa)=u_{\mathrm{infl}}(a)-\sqrt{\frac{1}{3}\left(g^{\prime}\left(u_{\mathrm{infl}}(a) ; a\right)-\kappa\right)}, \\
& u_{r}(\kappa)=u_{\mathrm{infl}}(a)+\sqrt{\frac{1}{3}\left(g^{\prime}\left(u_{\mathrm{infl}}(a) ; a\right)-\kappa\right)} .
\end{aligned}
$$

It is easy to verify that $u_{l}(\kappa)$ and $u_{r}(\kappa)$ are the two solutions to the quadratic equation $g^{\prime}(u ; a)=\kappa$. 
Lemma 3.8. Fix $0<a \leq \frac{1}{2}$ and $d>0$ and suppose that

$$
v_{\#}^{\prime}(u)=v_{d}^{\prime}(u)=\beta
$$

for some $\# \in\{-,+\}$ and $0 \leq u \leq a$, with $u \neq u_{\min }(a)$ in case $a=\frac{1}{2}$. Then the following statements hold.

(i) We have

$$
2 d\left[v_{\#}^{\prime \prime}(u)-v_{d}^{\prime \prime}(u)\right]=\frac{1}{1-\beta} g^{\prime \prime}(u ; a)+\frac{\beta^{3}}{1-\beta} g^{\prime \prime}\left(v_{\#}(u) ; a\right)
$$

(ii) If $\#=-$, then we have $\beta \in[-1,0) \cup(1, \infty)$. On the other hand, the inclusion $\beta \in(0,1)$ holds if $\#=+$.

(iii) We have $u=u_{l}(2 d(1-\beta))$.

(iv) Suppose that $\#=-$. Then the identity

$$
v_{\#}(u)=u_{l}\left(2 d\left(1-\beta^{-1}\right)\right)
$$

holds if $v_{-}(u) \leq u_{\mathrm{infl}}(a)$. On the other hand, we have

$$
v_{\#}(u)=u_{r}\left(2 d\left(1-\beta^{-1}\right)\right)
$$

if $v_{-}(u)>u_{\text {infl }}(a)$.

(v) The identity

$$
v_{\#}(u)=u_{r}\left(2 d\left(1-\beta^{-1}\right)\right)
$$

holds if $\#=+$.

Proof. We first consider the case $0<a<\frac{1}{2}$. For any $0 \leq \tilde{u} \leq a$, one sees that $v_{d}^{\prime}(\tilde{u})=1$ holds if and only if $g^{\prime}(\tilde{u} ; a)=v_{ \pm}^{\prime}(\tilde{u})=0$, which shows that $\beta \notin\{0,1\}$.

For any $0 \leq \tilde{u} \leq a$ we have $g^{\prime}\left(v_{+}(\tilde{u}) ; a\right)<0$, and hence

$$
\operatorname{sign}\left[v_{+}^{\prime}(\tilde{u})\right]=\operatorname{sign}\left[g^{\prime}(\tilde{u} ; a)\right] .
$$

If $\#=+$, this shows that $g^{\prime}(u ; a)>0$ and $v_{d}^{\prime}(u)>0$; hence $\beta \in(0,1)$.

On the other hand, we have $g^{\prime}\left(v_{-}(\tilde{u}) ; a\right)>0$ for all $0 \leq \tilde{u} \leq a$. If $g^{\prime}(u ; a)<0$, then we have $v_{d}^{\prime}(u)=\beta>1$, while if $g^{\prime}(u ; a)>0$ and $\#=-$, we may use item (v) from Lemma 3.6 to conclude

$$
-1 \leq v_{-}^{\prime}(u)=\beta<0
$$

which establishes (ii).

In order to obtain (iii), it suffices to recall the bound $u \leq a \leq u_{\text {infl }}(a)$ and note that the identity $v_{*}^{\prime}(u)=\beta$ implies that

$$
g^{\prime}(u)=2 d(1-\beta)
$$


On the other hand, the identity $v_{ \pm}^{\prime}(u)=\beta$ implies

$$
g^{\prime}\left(v_{ \pm}(u)\right)=-2 d \beta^{-1}(1-\beta) .
$$

Items (iv) and (v) now follow directly, remembering that $v_{+}(u) \geq u_{\max }(a) \geq u_{\text {infl }}(a)$.

Exploiting (3.53), we may now compute

$$
\begin{aligned}
v_{ \pm}^{\prime \prime}(u) & =\frac{\beta}{2 d(1-\beta)} g^{\prime \prime}(u ; a)+\frac{(2 d)^{2}(1-\beta)^{2} \beta^{3}}{(2 d)^{3}(1-\beta)^{3}} g^{\prime \prime}\left(v_{ \pm}(u) ; a\right) \\
& =\frac{\beta}{2 d(1-\beta)} g^{\prime \prime}(u ; a)+\frac{\beta^{3}}{2 d(1-\beta)} g^{\prime \prime}\left(v_{ \pm}(u) ; a\right) .
\end{aligned}
$$

The desired identity in (i) hence follows directly from (3.54). In order to conclude the proof, it suffices to note that the arguments above remain valid when $a=\frac{1}{2}$. Indeed, the critical cases $g^{\prime}(u ; a)=0$ and $g^{\prime}\left(v_{ \pm} ; a\right)=0$ are excluded by the requirement that $u \neq u_{\min }(a)$.

Lemma 3.9. Fix $0<a \leq \frac{1}{2}$, and suppose that

$$
v_{-}^{\prime}(u)=v_{d}^{\prime}(u)
$$

for some $u_{\min }(a) \leq u<a$ and $d>0$, with $u \neq u_{\min }(a)$ if $a=\frac{1}{2}$. Then we have

$$
v_{-}^{\prime \prime}(u)>v_{d}^{\prime \prime}(u) \text {. }
$$

Proof. Exploiting (v) of Lemma 3.6 and (ii) of Lemma 3.8, we have $v_{-}^{\prime}(u) \in(-1,0)$. In addition, the inequalities $v_{-}(u)>u$ and $g^{\prime \prime \prime}<0$ imply that $g^{\prime \prime}\left(v_{-}(u) ; a\right) \leq g^{\prime \prime}(u ; a)$. Writing $\beta=v_{-}^{\prime}(u)$, we may hence estimate

$$
\begin{aligned}
2 d\left[v_{-}^{\prime \prime}(u)-v_{d}^{\prime \prime}(u)\right] & \geq \frac{1}{1-\beta} g^{\prime \prime}(u ; a)+\frac{\beta^{3}}{1-\beta} g^{\prime \prime}(u ; a) \\
& =\frac{\beta^{3}+1}{1-\beta} g^{\prime \prime}(u ; a) \\
& >0,
\end{aligned}
$$

in which we used $g^{\prime \prime}(u ; a)>0$.

Intersections with $v_{ \pm}^{\prime}(u)>0$ are more delicate to analyze. Items (i), (iii), and (iv) of Lemma 3.8 suggest that it is worthwhile to consider the two functions

$$
\begin{aligned}
& h_{l}(\beta)=\frac{1}{2 d(1-\beta)}\left[g^{\prime \prime}\left(u_{l}(2 d(1-\beta)) ; a\right)+\beta^{3} g^{\prime \prime}\left(u_{l}\left(2 d\left(1-\beta^{-1}\right)\right) ; a\right)\right], \\
& h_{r}(\beta)=\frac{1}{2 d(1-\beta)}\left[g^{\prime \prime}\left(u_{l}(2 d(1-\beta)) ; a\right)+\beta^{3} g^{\prime \prime}\left(u_{r}\left(2 d\left(1-\beta^{-1}\right)\right) ; a\right)\right] .
\end{aligned}
$$

Lemma 3.10. Pick $0<a \leq \frac{1}{2}$ and $0<d \leq \frac{g^{\prime}(a ; a)}{4}$. Then for any $\beta>1$ the inequality

$$
h_{l}(\beta)<0
$$

holds, while for any $\beta \in(0, \infty) \backslash\{1\}$ we have

$$
h_{r}(\beta)>0 .
$$


Proof. Observe first that for $\beta>0$ we have

$$
\max \left\{2 d(1-\beta), 2 d\left(1-\beta^{-1}\right)\right\} \leq 2 d \leq \frac{g^{\prime}(a ; a)}{2} \leq \frac{g^{\prime}\left(u_{\text {inf }}(a) ; a\right)}{2},
$$

which implies that $h_{l}(\beta)$ and $h_{r}(\beta)$ are well defined.

A little algebra yields

$$
\begin{aligned}
& h_{l}(\beta)=\frac{\sqrt{3}}{d(1-\beta)}\left[\sqrt{g^{\prime}\left(u_{\mathrm{infl}}(a) ; a\right)+2 d(\beta-1)}+\beta^{3} \sqrt{g^{\prime}\left(u_{\mathrm{infl}}(a) ; a\right)+2 d \beta^{-1}(1-\beta)}\right], \\
& h_{r}(\beta)=\frac{\sqrt{3}}{d(1-\beta)}\left[\sqrt{g^{\prime}\left(u_{\mathrm{infl}}(a) ; a\right)+2 d(\beta-1)}-\beta^{3} \sqrt{g^{\prime}\left(u_{\mathrm{infl}}(a) ; a\right)+2 d \beta^{-1}(1-\beta)}\right] .
\end{aligned}
$$

It is clear that $h_{l}(\beta)<0$ for $\beta>1$. Upon writing

$$
\begin{aligned}
\Delta(\beta) & =g^{\prime}\left(u_{\mathrm{infl}}(a) ; a\right)+2 d(\beta-1)-\left(\beta^{6} g^{\prime}\left(u_{\mathrm{infl}}(a) ; a\right)+2 d \beta^{5}(1-\beta)\right) \\
& =\left(1-\beta^{6}\right)\left(g^{\prime}\left(u_{\mathrm{infl}}(a) ; a\right)-2 d\right)+2 d \beta\left(1-\beta^{4}\right),
\end{aligned}
$$

it is easy to verify that $\Delta(\beta)<0$ for $\beta>1$ and $\Delta(\beta)>0$ for $0<\beta<1$. This yields the final inequality (3.71).

Lemma 3.11. Pick $0<a \leq \frac{1}{2}$ and $0<d \leq \frac{g^{\prime}(a ; a)}{4}$. Then we have

$$
v_{-}^{\prime}(0)<v_{d}^{\prime}(0) \text {. }
$$

In addition, suppose that

$$
v_{-}^{\prime}(u)=v_{*}^{\prime}(u)
$$

for some $0 \leq u \leq a$, with $u \neq u_{\min }(a)$ if $a=\frac{1}{2}$. Then one of the following two statements must hold.

(a) We have the inequality

$$
v_{-}^{\prime \prime}(u)>v_{d}^{\prime \prime}(u)
$$

(b) We have the identities

$$
u=a, \quad d=\frac{g^{\prime}(a)}{4}, \quad v_{-}^{\prime \prime}(u)=v_{d}^{\prime \prime}(u) .
$$

Proof. An easy computation yields

$$
v_{d}^{\prime}(0)=1-\frac{g^{\prime}(0 ; a)}{2 d} \geq 1-\frac{2 g^{\prime}(0 ; a)}{g^{\prime}(a ; a)}>-\frac{g^{\prime}(0 ; a)}{g^{\prime}(a ; a)}=v_{-}^{\prime}(0) .
$$

We introduce the critical value

$$
u_{c}=\sup \left\{0 \leq u \leq a: v_{-}(\tilde{u}) \leq u_{\text {infl }}(a) \text { for all } 0 \leq \tilde{u} \leq u\right\}
$$

and remark that $u_{c}=0$ when $a=\frac{1}{2}$. This allows us to define the value

$$
u_{I}=\sup \left\{0 \leq u \leq \min \left\{u_{c}, u_{\min }(a)\right\}: v_{d}^{\prime}(u)>v_{-}^{\prime}(u)\right\},
$$


which again satisfies $u_{I}=0$ when $a=\frac{1}{2}$.

We claim that also $v_{d}^{\prime}\left(u_{I}\right)>v_{-}^{\prime}\left(u_{I}\right)$. Indeed, assuming this is false, we can define $\beta=$ $v_{d}^{\prime}\left(u_{I}\right)=v_{-}^{\prime}\left(u_{I}\right) \geq 0$. Item (ii) of Lemma 3.8 then implies $\beta>1$. Since $h_{l}(\beta)<0$, we must have

$$
v_{-}^{\prime \prime}\left(u_{I}\right)<v_{d}^{\prime \prime}\left(u_{I}\right)
$$

which yields a contradiction.

In particular, if (3.76) holds, then we must have $u \geq \min \left\{u_{\min }(a), u_{c}\right\}$. If $u_{\min }(a) \leq u<a$, Lemma 3.9 shows that (a) must hold. On the other hand, if $u_{c} \leq u<u_{\min }(a)$, then we can define $\beta=v_{d}^{\prime}(u)=v_{-}^{\prime}(u) \geq 0$ and conclude as above that $\beta>1$. In addition, we have $v_{-}(u) \geq u_{\text {infl }}(a)$, which allows us to use $h_{r}(\beta)>0$ and item (iv) of Lemma 3.8 to show that (a) must hold. In the final case $u=a$, the remarks at the start of this section together with a direct computation of $v_{d}^{\prime \prime}(a)$ and $v_{-}^{\prime \prime}(a)$ imply the identities in (b).

In the remainder of this section we collect several consequences of these computations. In each case, we either rule out nontransverse intersections of $v_{ \pm}$with $v_{d}$ or show that they must occur at local minima of $v_{ \pm}-v_{d}$.

Corollary 3.12. Fix $0<a \leq \frac{1}{2}$ together with $0<d \leq \frac{g^{\prime}(a ; a)}{4}$, and suppose that

$$
v_{+}^{\prime}(u)=v_{d}^{\prime}(u)
$$

for some $0 \leq u \leq a$, with $u \neq u_{\min }(a)$ if $a=\frac{1}{2}$. Then we have

$$
v_{+}^{\prime \prime}(u)>v_{d}^{\prime \prime}(u) .
$$

Proof. Using the fact that $h_{r}(\beta)>0$ for $\beta \in(0,1)$, this follows directly from items (i), (ii), and (v) of Lemma 3.8.

Corollary 3.13. Fix $0<a \leq \frac{1}{2}$ together with $0<d<\frac{g^{\prime}(a ; a)}{4}$, and suppose that

$$
v_{-}^{\prime}(u)=v_{*}^{\prime}(u)
$$

for some $0 \leq u \leq a$, with $u \neq u_{\min }(a)$ if $a=\frac{1}{2}$. Then we have

$$
v_{-}^{\prime \prime}(u)>v_{d}^{\prime \prime}(u) \text {. }
$$

In addition, we have

$$
v_{-}^{\prime}(a)>v_{d}^{\prime}(a) .
$$

Proof. The first inequality follows directly from the fact that option (b) in Lemma 3.11 cannot hold because of the restriction on $d$. The final inequality can be verified directly by noting that

$$
v_{d}^{\prime}(a)=1-\frac{g^{\prime}(a ; a)}{2 d}<1-\frac{2 g^{\prime}(a ; a)}{g^{\prime}(a ; a)}=-1=v_{-}^{\prime}(a) .
$$


Corollary 3.14. Fix $0<a \leq \frac{1}{2}$ together with $d=\frac{g^{\prime}(a ; a)}{4}$. Then we have

$$
v_{-}^{\prime}(u)<v_{d}^{\prime}(u)
$$

for all $0 \leq u<a$, with the exception of $u=u_{\min }(a)$ in the special case $a=\frac{1}{2}$.

Proof. It is easy to verify that $v_{-}^{\prime}(a)=v_{d}^{\prime}(a)$ and $v_{-}^{\prime \prime}(a)=v_{d}^{\prime \prime}(a)$. We also compute

$$
v_{d}^{\prime \prime \prime}(a)=-2 \frac{g^{\prime \prime \prime}(a ; a)}{g^{\prime}(a ; a)}=\frac{12}{a(1-a)}>0
$$

together with

$$
\begin{aligned}
v_{-}^{\prime \prime \prime}(u)=-\frac{g^{\prime \prime \prime}(u ; a)}{g^{\prime}\left(v_{-}(u) ; a\right)}-3 \frac{g^{\prime \prime}(u ; a) g^{\prime}(u ; a)}{g^{\prime}\left(v_{-}(u) ; a\right)^{3}} g^{\prime \prime}\left(v_{-}(u) ; a\right) & \\
& \quad-3 \frac{g^{\prime}(u ; a)^{3}}{g^{\prime}\left(v_{-}(u) ; a\right)^{5}} g^{\prime \prime}\left(v_{-}(u) ; a\right)^{2}+\frac{g^{\prime}(u ; a)^{3}}{g^{\prime}\left(v_{-}(u) ; a\right)^{4}} g^{\prime \prime \prime}\left(v_{-}(u) ; a\right),
\end{aligned}
$$

which gives

$$
v_{-}^{\prime \prime \prime}(a)=-6 \frac{g^{\prime \prime}(a ; a)^{2}}{g^{\prime}(a ; a)^{2}} \leq 0
$$

In particular, we see that

$$
v_{-}^{\prime}(a-\epsilon)<v_{d}^{\prime}(a-\epsilon)
$$

for all sufficiently small $\epsilon>0$. If $a \neq \frac{1}{2}$, the conclusion now follows from (3.75) together with (a) from Lemma 3.11.

For $a=\frac{1}{2}$, one also needs to use the identities

$$
v_{d}^{\prime}\left(u_{\min }(a)\right)=1, \quad v_{d}^{\prime \prime}\left(u_{\min }(a)\right)=-12 \sqrt{3}
$$

together with the limits in item (iv) of Lemma 3.7 to conclude that

$$
v_{-}^{\prime}\left(u_{\min }(a)-\epsilon\right)<v_{d}^{\prime}\left(u_{\min }(a)-\epsilon\right)
$$

for all sufficiently small $\epsilon>0$. The arguments above allow us to extend this to $\epsilon \in\left(0, u_{\min }(a)\right]$. In addition, we have $v_{-}^{\prime}(\tilde{u})=-1<v_{d}^{\prime}(\tilde{u})$ for $u_{\min }(a)<\tilde{u}<a$.

Corollary 3.15. Pick $0<a \leq \frac{1}{2}$ and $0<d \leq \frac{g^{\prime}(a ; a)}{4}$. Then we have

$$
v_{-}^{\prime}(u)<v_{d}^{\prime}(u)
$$

for all $0 \leq u<u_{\min }(a)$.

Proof. Writing $d_{c}=\frac{g^{\prime}(a ; a)}{4}$, we may use Corollary 3.14 to compute

$$
v_{d}^{\prime}(u)=1-\frac{1}{2 d} g^{\prime}(u ; a) \geq 1-\frac{2}{g^{\prime}(a ; a)} g^{\prime}(u ; a)=v_{d_{c}}^{\prime}(u)>v_{-}^{\prime}(u)
$$

for $u \in\left[0, u_{\min }(a)\right)$. 

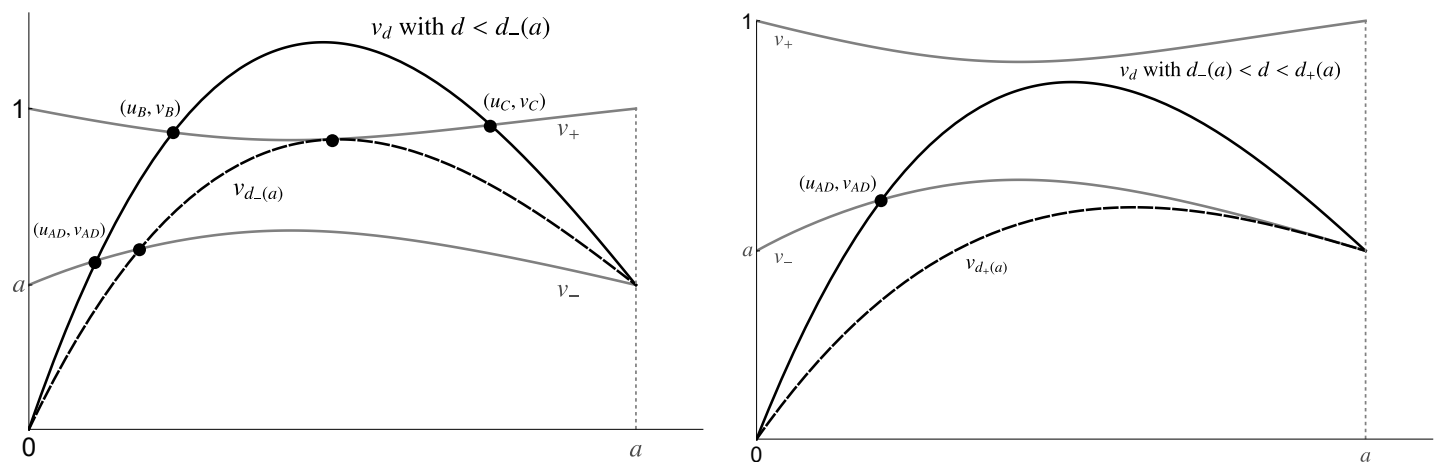

Figure 8. Fix $0<a<\frac{1}{2}$. The branches $u_{B}$ and $u_{C}$ described in Lemma 3.18 arise as the two intersections of $v_{+}$and $v_{d}$ on $[0, a]$, which collide as $d \uparrow d_{-}(a)$ (left). On the other hand, the branch $u_{A D}$ described in Lemma 3.17 arises as the unique intersection of the curves $v_{-}$and $v_{d}$ on $[0, a)$, which converges to a as $d_{\uparrow} d_{+}(a)$ (right).

Corollary 3.16. Fix $a=\frac{1}{2}$ and $0<d \leq \frac{g^{\prime}(a ; a)}{4}$. Then we have

$$
v_{+}^{\prime}(u)>v_{d}^{\prime}(u)
$$

for all $u_{\min }(a)<u \leq a$.

Proof. Item (iv) of Lemma 3.7 allows us to compute

$$
v_{d}^{\prime}\left(u_{\min }(a)\right)=1=\lim _{u \downarrow u_{\min }(a)} v_{+}^{\prime}(u)
$$

together with

$$
v_{d}^{\prime \prime}\left(u_{\min }(a)\right)<\lim _{u \downarrow u_{\min }(a)} v_{+}^{\prime \prime}(u),
$$

which allows us to conclude that

$$
v_{+}^{\prime}\left(u_{\min }(a)+\epsilon\right)>v_{d}^{\prime}\left(u_{\min }(a)+\epsilon\right)
$$

for all sufficiently small $\epsilon>0$. Corollary 3.12 allows us to extend this conclusion to the desired interval $\epsilon \in\left(0, a-u_{\min }(a)\right)$.

3.3. Structure. We are now ready to analyze the global structure of the solution set to $G(u, v ; a, d)=0$. Our first two results fix $a \in\left(0, \frac{1}{2}\right]$ and track the intersections of the curves $v_{ \pm}$that were introduced in section 3.1 with the curve $v_{d}$ introduced in section 3.2. These intersections disappear as the parameter $d$ is increased; see Figure 8.

Lemma 3.17. Fix $0<a \leq \frac{1}{2}$. Then there exists a continuous strictly increasing function

$$
u_{A D}:\left[0, \frac{g^{\prime}(a ; a)}{4}\right] \rightarrow[0, a]
$$

that satisfies the following properties.

(i) We have $u_{A D}(0)=0$ and $u_{A D}\left(\frac{g^{\prime}(a ; a)}{4}\right)=a$. 
(ii) The identity $v_{-}\left(u_{A D}(d)\right)=v_{d}\left(u_{A D}(d)\right)$ holds for any $0<d \leq \frac{g^{\prime}(a ; a)}{4}$.

(iii) Suppose that $v_{-}(u)=v_{d}(u)$ for some $0<d \leq \frac{g^{\prime}(a ; a)}{4}$ and $0 \leq u \leq a$. Then in fact $u \in\left\{u_{A D}(d), a\right\}$.

(iv) Consider any $0<d<\frac{g^{\prime}(a ; a)}{4}$ for which $(a, d) \neq\left(\frac{1}{2}, \frac{1}{24}\right)$. Then we have the inequality

$$
v_{-}^{\prime}\left(u_{A D}(d)\right)<v_{d}^{\prime}\left(u_{A D}(d)\right) .
$$

(v) For any $d>\frac{g^{\prime}(a ; a)}{4}$ and $0 \leq u<a$ we have $v_{-}(u)>v_{d}(u)$.

Proof. For convenience, we introduce the function $h_{d}(u)=v_{-}(u)-v_{d}(u)$ and set out to count the zeros of $h_{d}$ on the interval [0,a]. We first note that $h_{d}(a)=0$ for all $d>0$. When $d=\frac{g^{\prime}(a ; a)}{4}$, this is in fact the only zero, which can be seen by using (3.89) and explicitly verifying the inequality

$$
v_{-}\left(u_{\min }(a)\right)>v_{d}\left(u_{\min }(a)\right)
$$

for $(a, d)=\left(\frac{1}{2}, \frac{g^{\prime}\left(\frac{1}{2} ; \frac{1}{2}\right)}{4}\right)$.

On the other hand, (3.87) implies that $h_{d}$ has at least two zeros for $0<d<\frac{g^{\prime}(a ; a)}{4}$. Furthermore, we claim that $h_{d}$ has precisely two zeros for $0<d \leq d_{1}$ upon choosing $d_{1}>0$ to be sufficiently small. Indeed, for any $u \in(0, a)$ we have $v_{d}^{\prime \prime}(u)<0$, and we can enforce

$$
\left|v_{d}^{\prime}(u)\right|+v_{d}(u) \geq 1+\max _{0 \leq \tilde{u} \leq a}\left\{\left|v_{-}^{\prime}(\tilde{u})\right|\right\}
$$

by restricting the size of $d>0$.

We now pick $\epsilon>0$ in such a way that the function $h_{d}$ is strictly decreasing on $\left[0, u_{\min }(a)+\epsilon\right]$ for all $d_{1} \leq d \leq \frac{g^{\prime}(a ; a)}{4}$. This is possible because Corollary 3.15 allows us to enforce $h_{d}^{\prime}<0$ on this interval, with the exception of the single point $u=u_{\min }(a)$ when $a=\frac{1}{2}$.

Let us now define the critical value

$$
d_{c}=\sup \left\{d_{1} \leq d \leq \frac{g^{\prime}(a ; a)}{4}: h_{d}=0 \text { has two distinct solutions on }[0, a]\right\}
$$

and assume for the moment that $d_{c}<g^{\prime}(a ; a) / 4$. The preparations above show that there exists $u_{\min }(a)<u<a$ with $h_{d_{c}}(u)=0$ and $h_{d_{c}}^{\prime}(u)=0$. Corollary 3.13 now implies $h_{d_{c}}^{\prime \prime}(u)>$ 0 . As a consequence of the monotonicity $\partial_{d} v_{d}(u)<0$, this means that for all sufficiently small $\delta>0$, the function $h_{d}$ with $d=d_{c}-\delta$ must have at least three zeros. This yields a contradiction, which implies that $d_{c}=g^{\prime}(a ; a) / 4$.

We may hence define $u_{A D}(d) \in[0, a]$ to be the leftmost root of $h_{d}(u)=0$ for $0<d \leq$ $d_{c}$. The statements (i)-(v) follow readily from the observations above together with the monotonicity $\partial_{d} v_{d}(u)<0$ that holds for $u \in(0, a)$.

Lemma 3.18. Fix $0<a \leq \frac{1}{2}$. Then there exists a constant $0<d_{-}<\frac{g^{\prime}(a ; a)}{4}$ together with two continuous functions

$$
\left(u_{B}, u_{C}\right):\left[0, d_{-}\right] \rightarrow[0, a] \times[0, a]
$$

that satisfy the following properties. 
(i) We have $\left(u_{B}, u_{C}\right)(0)=(0, a)$ and $u_{B}\left(d_{-}\right)=u_{C}\left(d_{-}\right)$.

(ii) The function $u_{B}$ is strictly increasing, while the function $u_{C}$ is strictly decreasing.

(iii) For any $0<d \leq d_{-}$the identity $v_{+}\left(u_{\#}\right)=v_{d}\left(u_{\#}\right)$ holds for $\# \in\{B, C\}$.

(iv) If $v_{+}(u)=v_{d}(u)$ for some $0<d \leq d_{-}$and $0 \leq u \leq a$, then in fact $u \in\left\{u_{B}(d), u_{C}(d)\right\}$.

(v) For any $0<d<d_{-}$we have

$$
v_{+}^{\prime}\left(u_{B}(d)\right)<v_{d}^{\prime}\left(u_{B}(d)\right), \quad v_{+}^{\prime}\left(u_{C}(d)\right)>v_{d}^{\prime}\left(u_{C}(d)\right) .
$$

If $a \neq \frac{1}{2}$, then we also have

$$
v_{+}^{\prime}\left(u_{C}\left(d_{-}\right)\right)=v_{+}^{\prime}\left(u_{B}\left(d_{-}\right)\right)=v_{d}^{\prime}\left(u_{B}\left(d_{-}\right)\right)=v_{d}^{\prime}\left(u_{C}\left(d_{-}\right)\right) .
$$

(vi) For any $d>d_{-}$the inequality $v_{+}(u)>v_{d}(u)$ holds for all $0 \leq u \leq a$.

(vii) If $a=\frac{1}{2}$, then we have $d_{-}\left(\frac{1}{2}\right)=\frac{1}{24}$ together with $u_{B}\left(\frac{1}{24}\right)=u_{C}\left(\frac{1}{24}\right)=u_{\min }(a)$.

Proof. Writing $h_{d}(u)=v_{+}(u)-v_{d}(u)$, we observe first that $h_{d}$ is strictly decreasing on $\left[0, u_{\min }(a)\right]$ because $v_{d}^{\prime}>0$ and $v_{+}^{\prime}<0$ on the interior of this interval.

In addition, in the special case $a=\frac{1}{2}$, we can use Corollary 3.16 to conclude that $h_{d}$ is strictly increasing on $\left[u_{\min }(a), a\right]$. Since $h_{d}\left(u_{\min }(a)\right)=0$ occurs precisely when $d=\frac{1}{24}$, all the desired statements can be easily verified.

Throughout the remainder of this proof we therefore assume that $0<a<\frac{1}{2}$. Arguing as in the proof of Lemma 3.17, we may pick $d_{1}>0$ in such a way that $h_{d}$ has precisely two zeros on $[0, a]$ for every $0<d \leq d_{1}$. This allows us to define the critical value

$$
d_{-}=\sup \left\{d_{1} \leq d: h_{d}=0 \text { has two distinct solutions on }[0, a]\right\} .
$$

Since $v_{-}<v_{+}$, it is clear that $d_{-}<\frac{g^{\prime}(a ; a)}{4}$.

Let us assume for the moment that $h_{d_{-}}$has two or more zeros on $[0, a]$. This implies that there exists at least one $u \in(0, a)$ for which $h_{d_{-}}(u)=0$ and $h_{d_{-}}^{\prime}(u)=0$. Using Corollary 3.13 it follows that $h_{d_{-}}^{\prime \prime}(u)>0$ must hold for all such zeros. As a consequence of the monotonicity $\partial_{d} v_{d}(u)<0$, this means that for all sufficiently small $\delta>0$, the function $h_{d}$ with $d=d_{-}-\delta$ must have at least three zeros. This yields a contradiction, which by continuity shows that $h_{d_{-}}(u)=0$ has precisely one root on $[0, a]$. Upon defining $u_{B}(d)$ and $u_{C}(d)$ to be the leftmost, respectively, rightmost root of $h_{d}(u)=0$, the desired properties (i)-(vii) can be easily verified.

Recalling the function $G$ introduced in (2.6), we see that

$$
D_{1,2} G(u, v ; a, d)=\left(\begin{array}{cc}
g^{\prime}(u)-2 d & 2 d \\
2 d & g^{\prime}(v)-2 d
\end{array}\right) .
$$

In order to study the stability and parameter-dependence of the roots constructed in Lemmas $3.17-3.18$, it is crucial to understand when the determinant of $D_{1,2} G$ vanishes. The result below states that this happens at tangential intersections of $v_{ \pm}$and $v_{d}$.

Lemma 3.19. Fix $0<a<\frac{1}{2}$ together with $d>0$, and suppose that

$$
v_{\#}(u)=v_{d}(u)
$$


for some $\# \in\{-,+\}$ and $u \in[0, a]$. Then we have $\operatorname{det} D_{1,2} G\left(u, v_{d}(u) ; a, d\right)=0$ if and only if $v_{\#}^{\prime}(u)=v_{d}^{\prime}(u)$.

Proof. We first consider the case $v_{\#}^{\prime}(u)=0$, which occurs when $g^{\prime}(u ; a)=0$ and hence $u=u_{\min }(a)$. Using $0<a<\frac{1}{2}$, we see that

$$
v_{-}(u)<u_{\max }(a)<v_{+}(u) .
$$

Writing $v=v_{d}(u)=v_{ \pm}(u)$, we hence obtain $g^{\prime}(v ; a) \neq 0$, and hence

$$
\operatorname{det} D_{1,2} G(u, v ; a, d)=-2 d g^{\prime}(v ; a) \neq 0 .
$$

Since $v_{d}^{\prime}(u)=1$, the desired equivalence indeed holds for this case.

Assuming now that $v_{\#}^{\prime}(u) \neq 0$ and hence $g^{\prime}(u ; a) \neq 0$, we again write $v=v_{d}(u)=v_{\#}(u)$ and use (3.34) to compute

$$
g^{\prime}(v ; a)=-\left[v_{\#}^{\prime}(u)\right]^{-1} g^{\prime}(u ; a) .
$$

In particular, we find

$$
\begin{aligned}
\operatorname{det} D_{1,2} G(u, v ; a, d) & =\left(g^{\prime}(u ; a)-2 d\right)\left(-\left[v_{\#}^{\prime}(u)\right]^{-1} g^{\prime}(u ; a)-2 d\right)-4 d^{2} \\
& =-\left[v_{\#}^{\prime}(u)\right]^{-1} g^{\prime}(u ; a)^{2}-2 d g^{\prime}(u ; a)+2 d\left[v_{\#}^{\prime}(u)\right]^{-1} g^{\prime}(u ; a) \\
& =2 d g^{\prime}(u ; a)\left[v_{\#}^{\prime}(u)\right]^{-1}\left[1-\frac{g^{\prime}(u ; a)}{2 d}-v_{\#}^{\prime}(u)\right] \\
& =2 d g^{\prime}(u ; a)\left[v_{\#}^{\prime}(u)\right]^{-1}\left[v_{d}^{\prime}(u)-v_{\#}^{\prime}(u)\right],
\end{aligned}
$$

from which the statement follows.

In order to characterize the dependence of $d_{-}(a)$ on $a$, we introduce the function

$$
G_{\mathrm{sn}}(u, v, d ; a)=\left(G_{1}(u, v ; a, d), G_{2}(u, v ; a, d), \operatorname{det} D_{1,2} G(u, v ; a, d)\right)^{T}
$$

and symbolically write

$$
\left[D_{1,2,3} G_{\mathrm{sn}}(u, v, d ; a)\right]^{-1}=\left[\operatorname{det} D_{1,2,3} G_{\mathrm{sn}}(u, v, d ; a)\right]^{-1}\left(\begin{array}{ccc}
* & * & * \\
* & * & * \\
\gamma_{1}(u, v, d ; a) & \gamma_{2}(u, v, d ; a) & \gamma_{3}(u, v, d ; a)
\end{array}\right) .
$$

For any $0<a \leq \frac{1}{2}$, we use the functions defined in Lemma 3.18 to introduce the notation

$$
\omega(a)=\left(u_{B}\left(d_{-}(a)\right), v_{+}\left(u_{B}\left(d_{-}(a)\right)\right), d_{-}(a)\right)=\left(u_{C}\left(d_{-}(a)\right), v_{+}\left(u_{C}\left(d_{-}(a)\right)\right), d_{-}(a)\right)
$$

which corresponds to the critical point where the branches $u_{B}$ and $u_{C}$ collide.

Corollary 3.20. Upon fixing $0<a<\frac{1}{2}$, the following two statements are equivalent.

(a) The identity $G_{\mathrm{sn}}(u, v, d ; a)=0$ holds for some $d>0$ and some pair $(u, v) \in[0,1]^{2}$ that has $v \geq u$. 
(b) We have $(u, v, d)=\omega(a)$ or $(u, v, d)=\left(a, a, \frac{g^{\prime}(a ; a)}{4}\right)$.

Proof. As preparation, we note that $\operatorname{det} D_{1,2} G(a, a ; a, d)=0$ if and only if $d=\frac{g^{\prime}(a ; a)}{4}$. In addition, it is easy to check that $\operatorname{det} D_{1,2} G(0,0 ; a, d)>0$ and $\operatorname{det} D_{1,2} G(1,1 ; a, d)>0$ for all $d \geq 0$.

The implication (b) $\rightarrow$ (a) can now be verified directly using Lemma 3.19 and (3.109). In addition, we only need to establish the reverse implication under the additional assumption that $v>u$.

Let us therefore assume that (a) holds with $g(u)=-g(v) \neq 0$, which allows us to write

$$
0<u<a<v<1 \text {. }
$$

In particular, Lemma 3.6 implies that $v=v_{-}(u)$ or $v=v_{+}(u)$. Lemma 3.19 together with (3.108) and (3.103) now imply that (b) must hold.

Lemma 3.21. We have the identities

$$
\begin{aligned}
& \gamma_{1}(0,1,0 ; 0)=-2, \\
& \gamma_{2}(0,1,0 ; 0)=0 .
\end{aligned}
$$

In addition, the identity

$$
\gamma_{3}(\omega(a) ; a)=0
$$

holds for any $0<a<\frac{1}{2}$, together with the inequalities

$$
\begin{aligned}
& \operatorname{det} D_{1,2,3} G_{\mathrm{sn}}(\omega(a) ; a) \quad<0, \\
& \gamma_{1}(\omega(a) ; a)-\gamma_{2}(\omega(a) ; a)<0, \\
& \gamma_{1}(\omega(a) ; a) \gamma_{2}(\omega(a) ; a) \geq 0 .
\end{aligned}
$$

Proof. Let us assume for the moment that $G_{\mathrm{sn}}(u, v, d ; a)=0$, which directly implies

$$
\left(g^{\prime}(u ; a)-2 d\right) g^{\prime}(v ; a)=2 d g^{\prime}(u ; a) .
$$

In view of the identity

$$
D_{1,2,3} G_{\mathrm{sn}}(u, v, d ; a)=\left(\begin{array}{ccc}
g^{\prime}(u ; a)-2 d & 2 d & 2(v-u) \\
2 d & g^{\prime}(v ; a)-2 d & 2(u-v) \\
\left(g^{\prime}(v ; a)-2 d\right) g^{\prime \prime}(u ; a) & \left(g^{\prime}(u ; a)-2 d\right) g^{\prime \prime}(v ; a) & -2\left(g^{\prime}(u ; a)+g^{\prime}(v ; a)\right)
\end{array}\right),
$$

we can use (3.124) to compute

$$
\begin{aligned}
\operatorname{det} D_{1,2,3} G_{\mathrm{sn}}(u, v, d ; a) & =2(v-u)\left[g^{\prime}(u ; a)\left(g^{\prime}(u ; a)-2 d\right) g^{\prime \prime}(v ; a)-g^{\prime}(v ; a)\left(g^{\prime}(v ; a)-2 d\right) g^{\prime \prime}(u ; a)\right] \\
& =2(v-u)\left[2 d g^{\prime}(u ; a)^{2} \frac{1}{g^{\prime}(v ; a)} g^{\prime \prime}(v ; a)-g^{\prime}(v ; a)\left(g^{\prime}(v ; a)-2 d\right) g^{\prime \prime}(u ; a)\right] .
\end{aligned}
$$


Expanding the subdeterminants of (3.125) and reusing (3.124), we also find

$$
\begin{aligned}
& \gamma_{1}(u, v, d ; a)=2 d\left(g^{\prime}(u ; a)-2 d\right) g^{\prime \prime}(v ; a)-\left(g^{\prime}(v ; a)-2 d\right)^{2} g^{\prime \prime}(u ; a), \\
& \gamma_{2}(u, v, d ; a)=2 d\left(g^{\prime}(v ; a)-2 d\right) g^{\prime \prime}(u ; a)-\left(g^{\prime}(u ; a)-2 d\right)^{2} g^{\prime \prime}(v ; a), \\
& \gamma_{3}(u, v, d ; a)=0
\end{aligned}
$$

which allows us to explicitly verify (3.121).

Let us now fix $0<a<\frac{1}{2}$. For any $(u, v) \in[0,1]^{2}$ and $d>0$ we introduce the function

$$
\begin{aligned}
h(u, v, d) & =\frac{1}{2 d} g^{\prime \prime}(u ; a)-\frac{g^{\prime \prime}(u ; a)}{g^{\prime}(v ; a)}-\frac{g^{\prime}(u ; a)^{2}}{g^{\prime}(v ; a)^{3}} g^{\prime \prime}(v ; a) \\
& =\frac{1}{2 d g^{\prime}(v ; a)^{2}}\left[g^{\prime}(v ; a)\left(g^{\prime}(v ; a)-2 d\right) g^{\prime \prime}(u ; a)-2 d \frac{g^{\prime}(u ; a)^{2}}{g^{\prime}(v ; a)} g^{\prime \prime}(v ; a)\right],
\end{aligned}
$$

which allows us to rewrite (3.126) in the form

$$
\operatorname{det} D_{1,2,3} G_{\mathrm{sn}}(u, v, d ; a)=-4 d(v-u) g^{\prime}(v ; a)^{2} h(u, v, d) .
$$

Using (3.53) and (3.54), we observe that

$$
v_{+}^{\prime \prime}(u)-v_{d}^{\prime \prime}(u)=h\left(u, v_{+}(u), d\right) .
$$

In particular, Corollary 3.12 and (3.109) imply that

$$
h(\omega(a))>0,
$$

which shows that

$$
\operatorname{det} D_{1,2,3} G_{\mathrm{sn}}(\omega(a) ; a)<0,
$$

as desired.

Let us now write $(u, v, d)=\omega(a)$ and compute

$$
\begin{aligned}
\gamma_{1}(\omega(a) ; a)-\gamma_{2}(\omega(a) ; a) & =g^{\prime}(u)\left(g^{\prime}(u)-2 d\right) g^{\prime \prime}(v)-g^{\prime}(v)\left(g^{\prime}(v)-2 d\right) g^{\prime \prime}(u) \\
& =\frac{1}{2}(v-u)^{-1} \operatorname{det} D G_{\mathrm{sn}}(u, v, d ; a) \\
& \leq 0 .
\end{aligned}
$$

In addition, since $u>u_{\text {min }}(a)$ and hence $v_{d}^{\prime}(u)=v_{+}^{\prime}(u)>0$, we have $g^{\prime}(u)<2 d$. This allows us to define

$$
\begin{aligned}
& \alpha=\sqrt{2 d}\left(2 d-g^{\prime}(u ; a)\right)^{3 / 2} g^{\prime \prime}(v ; a) \\
& \beta=\sqrt{2 d}\left(2 d-g^{\prime}(v ; a)\right)^{3 / 2} g^{\prime \prime}(u ; a)
\end{aligned}
$$

and compute

$$
\begin{aligned}
\gamma_{1}(\omega(a) ; a) \gamma_{2}(\omega(a) ; a)= & \left(4 d^{2}\right)\left(4 d^{2}\right) g^{\prime \prime}(u ; a) g^{\prime \prime}(v ; a)+\left(4 d^{2}\right)^{2} g^{\prime \prime}(u ; a) g^{\prime \prime}(v ; a) \\
& \quad-2 d\left(g^{\prime}(u ; a)-2 d\right)^{3} g^{\prime \prime}(v ; a)^{2}-2 d\left(g^{\prime}(v ; a)-2 d\right)^{3} g^{\prime \prime}(u ; a)^{2} \\
= & 2 \alpha \beta+\alpha^{2}+\beta^{2} \\
\geq & 0,
\end{aligned}
$$

as desired. 
Corollary 3.22. The map $a \mapsto \omega(a)$ is $C^{\infty}$-smooth on $\left(0, \frac{1}{2}\right)$, and we have $d_{-}^{\prime}(a)>0$.

Proof. Since $d_{-}<\frac{g^{\prime}(a ; a)}{4}$, we may use Corollary 3.20, together with the first inequality in (3.123), to apply the implicit function and establish the $C^{\infty}$-smoothness of $\omega$.

Since $g^{\prime}(a ; a) / 4 \downarrow 0$ as $a \downarrow 0$, a squeezing argument shows that $\lim _{a \downarrow 0} \omega(a)=(0,1,0)$. Exploiting (3.121) and (3.123), the continuity of $\gamma_{1}$ and $\gamma_{2}$ implies that

$$
\gamma_{1}(\omega(a) ; a)<\gamma_{2}(\omega(a) ; a) \leq 0
$$

for all $0<a<\frac{1}{2}$. In particular, writing $(u, v, d)=\omega(a)$, we may use the inequalities

$$
D_{2} g(u ; a)<0, \quad D_{2} g(v ; a)<0
$$

to compute

$$
d_{-}^{\prime}(a)=-\left[\operatorname{det} D_{1,2,3} G_{\mathrm{sn}}(\omega(a) ; a)\right]^{-1}\left[\gamma_{1}(\omega(a) ; a) D_{2} g(u ; a)+\gamma_{2}(\omega(a) ; a) D_{2} g(v ; a)\right]>0 .
$$

Proof of Proposition 3.1. Items (i), (iii), and (iv) follow from Lemmas 3.17-3.18 and the observations in the proof of Corollary 3.20. Item (ii) follows from Corollary 3.22, while the expansions in (v) follow from Propositions SM1.1 and SM1.4 in the supplementary material file M118922_01.pdf [local/web 255KB].

Proof of Proposition 3.2. The identity $g(1-u, 1-a)=-g(u, a)$ implies that

$$
G(1-u, 1-v ; 1-a, d)=-G(u, v ; a, d) .
$$

This allows us to extend the solutions constructed in Lemmas 3.17-3.18 to the entire interval $0 \leq a \leq 1$ in the fashion outlined in Corollary 3.4, which yields (ii) and (iv).

To establish (i), we note that for each \# $\in\{A, B, C\}$ the $\operatorname{sign}$ of $\operatorname{det} D_{1,2} G\left(\bar{u}_{\#}, \bar{v}_{\#} ; a, d\right)$ is constant on $\Omega_{-}$on account of Corollary 3.20. Using (ii) the eigenvalues of these matrices can be explicitly computed at $d=0$, which yields the desired stability properties.

To obtain the strict ordering in (iii), we fix $0<a \leq \frac{1}{2}$ and $0<d<d_{-}(a)$. We observe that $v_{+}(u) \geq v_{-}(u)$ for $u \in[0, a]$, with equality only at $u=u_{\min }(a)$ for $a=\frac{1}{2}$. Since $v_{d}(0)=0$, this implies that $\bar{u}_{A}(a, d)<\bar{u}_{B}(a, d)$ and $\bar{v}_{A}(a, d)<\bar{v}_{B}(a, d)$. By construction, we also have $\bar{u}_{B}(a, d)<\bar{u}_{C}(a, d)$. In addition, it cannot be the case that $\bar{v}_{B}(a, \tilde{d})=\bar{v}_{C}(a, \tilde{d})$ for any $0<\tilde{d}<d_{-}(a)$ since then $G_{2}=0$ implies that also $\bar{u}_{B}(a, \tilde{d})=\bar{u}_{C}(a, \tilde{d})$. Using the general identity

$$
D_{a, d}\left(\bar{u}_{\#}, \bar{v}_{\#}\right)=-\left[D_{1,2} G\left(\bar{u}_{\#}, \bar{v}_{\#} ; a, d\right)\right]^{-1} D_{3,4} G\left(\bar{u}_{\#}, \bar{v}_{\#} ; a, d\right),
$$

we see that

$$
\partial_{d} \bar{v}_{\#}(a, 0)=-\frac{1}{g^{\prime}\left(\bar{v}_{\#}(a, 0) ; a\right)}\left(\bar{u}_{\#}(a, 0)-\bar{v}_{\#}(a, 0)\right),
$$

which yields

$$
\partial_{d} \bar{v}_{B}(a, 0)=\frac{1}{g^{\prime}(1 ; a)}<\frac{1-a}{g^{\prime}(1 ; a)}=\partial_{d} \bar{v}_{C}(a, 0) .
$$

These observations allow us to conclude $\bar{v}_{B}(a, d)<\bar{v}_{C}(a, d)$, as desired.

Proof of Proposition 3.3. Arguing in a similar fashion as the proof of Proposition 3.2, the statements follow from Lemma 3.17 and Corollary 3.20. 
4. Travelling waves. Our goal here is to establish the existence of bichromatic wave solutions to the Nagumo LDE (2.1) and to obtain detailed results concerning their speed. In particular, we establish Theorems 2.2 and 2.3, which are the main results of this paper. The general ideas developed in this section can also be applied to other bistable nonlinearities $g$, but the quantitative results will naturally differ.

Upon introducing the standard discrete Laplacian

$$
\left[\Delta^{+} u\right](\xi)=u(\xi+1)+u(\xi-1)-2 u(\xi)
$$

together with the off-diagonal matrix

$$
\mathcal{J}=\left(\begin{array}{ll}
0 & 1 \\
1 & 0
\end{array}\right)
$$

the travelling wave system (2.5) can be rewritten as

$$
-c \Phi^{\prime}=d \mathcal{J} \Delta^{+} \Phi+G(\Phi ; a, d) .
$$

For any $(a, d) \in \Omega_{-}$, we seek solutions to (4.3) that satisfy the boundary conditions

$$
\lim _{\xi \rightarrow-\infty} \Phi(\xi)=(0,0), \quad \lim _{\xi \rightarrow+\infty} \Phi(\xi)=\left(\bar{u}_{B}(a, d), \bar{v}_{B}(a, d)\right) .
$$

The existence of such solutions will be established in section 4.1, where we also show $c \geq 0$ and establish Theorem 2.2. In section 4.2 we subsequently set out to derive criteria that distinguish between the two cases $c=0$ and $c>0$. We verify these criteria in section 4.3 for parameters $(a, d) \in \Omega_{-}$that are close to the cusp $\left(\frac{1}{2}, \frac{1}{24}\right)$ and the corner $(1,0)$. This allows us to establish our main results contained in Theorem 2.3 .

4.1. Existence of waves. Our preparatory work in section 3 allows us to invoke the theory developed in [11] to establish a general existence result for bichromatic waves. Indeed, the equilibria $(0,0)$ and $\left(\bar{u}_{B}, \bar{v}_{B}\right)$ are both stable under the dynamics of $(\dot{u}, \dot{v})=G(u, v ; a, d)$ and all intermediate equilibria are unstable. Using a straightforward estimate based on the ordering (3.11), the wavespeeds can be shown to be nonnegative.

Lemma 4.1. Pick $(a, d) \in \Omega_{-}$. Then there exists a constant $c \in \mathbb{R}$ and a nondecreasing function $\Phi: \mathbb{R} \rightarrow \mathbb{R}^{2}$ that satisfy (4.3)-(4.4). This constant $c$ is unique, while the function $\Phi$ is unique up to translation if $c \neq 0$. In the latter case we also have $\Phi^{\prime}(\xi)>(0,0)$ for all $\xi \in \mathbb{R}$.

Proof. In order to apply the theory from [11], it suffices to linearly stretch the variables $(u, v)$ in such a way that $\left(\bar{u}_{B}, \bar{v}_{B}\right)$ is mapped to $(1,1)$. Indeed, the assumptions (A1)-(A5) in [11, section 1] with $n=2$ can be verified by a simple inspection of the LDE (2.1). On the other hand, condition (BS) in [11, section 8] is satisfied on account of the discussion above concerning the stability of the 2-periodic equilibria of this LDE. The existence of the pair $(c, \Phi)$ now follows directly from [11, Thm. 6]. In addition, the monotonicity properties can be read off from [11, Thm. 5], while the uniqueness claims were established in [11, Thm. 3].

Lemma 4.2. Pick $(a, d) \in \Omega_{-}$. Then the constant $c$ defined in Lemma 4.1 satisfies $c \geq 0$. 
Proof. Suppose that $c \neq 0$. We estimate

$$
\begin{aligned}
-c \Phi_{v}^{\prime}(\xi) & =d\left[\Phi_{u}(\xi+1)+\Phi_{u}(\xi-1)-2 \Phi_{v}(\xi)\right]+g\left(\Phi_{v}(\xi) ; a\right) \\
& \leq 2 d[\bar{u}-v(\xi)]+g\left(\Phi_{v}(\xi) ; a\right) .
\end{aligned}
$$

Since $0<\bar{u}_{B}(a, d)<a<\bar{v}_{B}(a, d)$, there exists $\xi_{*} \in \mathbb{R}$ for which $\Phi_{v}\left(\xi_{*}\right)=\bar{u}_{B}(a, d)$. This yields

$$
-c \Phi_{v}^{\prime}\left(\xi_{*}\right) \leq g(\bar{u} ; a)<0
$$

from which we conclude $c>0$.

Writing $c(a, d)$ and $\Phi(a, d)$ for the wavespeed and waveprofile defined in Lemma 4.1, we introduce the set

$$
\mathcal{T}=\left\{(a, d) \in \Omega_{-}: c(a, d)>0\right\}
$$

which corresponds to the set $\mathcal{T}_{\text {low }}$ used in section 2 . In addition, for any $(a, d) \in \mathcal{T}$ we introduce the linear operators

$$
\mathcal{L}_{a, d}: W^{1, \infty}\left(\mathbb{R} ; \mathbb{R}^{2}\right) \rightarrow L^{\infty}\left(\mathbb{R} ; \mathbb{R}^{2}\right), \quad \mathcal{L}_{a, d}^{\text {adj }}: W^{1, \infty}\left(\mathbb{R} ; \mathbb{R}^{2}\right) \rightarrow L^{\infty}\left(\mathbb{R} ; \mathbb{R}^{2}\right),
$$

which act as

$$
\begin{aligned}
& \mathcal{L}_{a, d} \phi=-c(a, d) \phi^{\prime}-d \mathcal{J} \Delta^{+} \phi-D G(\Phi(a, d) ; a, d) \phi, \\
& \mathcal{L}_{a, d}^{\text {adj }} \psi=c(a, d) \psi^{\prime}-d \mathcal{J} \Delta^{+} \psi-D G(\Phi(a, d) ; a, d) \psi .
\end{aligned}
$$

The results in $\left[28\right.$, section 8] imply that there exists $\Psi=\Psi(a, d) \in W^{1, \infty}\left(\mathbb{R} ; \mathbb{R}^{2}\right)$ with $\Psi>$ $(0,0)$ for which we have the identities

$$
\operatorname{Ker} \mathcal{L}_{a, d}=\operatorname{span}\left\{\Phi^{\prime}(a, d)\right\}, \quad \operatorname{Ker} \mathcal{L}_{a, d}^{\text {adj }}=\operatorname{span}\{\Psi(a, d)\}
$$

together with the normalization

$$
\int_{-\infty}^{\infty}\left\langle\Psi(\xi), \Phi^{\prime}(\xi)\right\rangle d \xi=1
$$

In particular, [32, Thm. A] implies that

$$
\text { Range } \mathcal{L}_{a, d}=\left\{f \in L^{\infty}\left(\mathbb{R} ; \mathbb{R}^{2}\right): \int_{-\infty}^{\infty}\langle\Psi(\xi), f(\xi)\rangle d \xi=0\right\} .
$$

These ingredients allow us to use the implicit function theorem to show that the pair $(c, \Phi)$ depends smoothly on the parameters $(a, d) \in \mathcal{T}$. In addition, we obtain a sign on $\partial_{a} c$. 
Lemma 4.3. The maps

$$
\begin{aligned}
& \mathcal{T} \ni(a, d) \mapsto c(a, d) \quad \in(0, \infty), \\
& \mathcal{T} \ni(a, d) \quad \mapsto \quad \Phi(a, d) \quad \in \quad B C^{1}\left(\mathbb{R} ; \mathbb{R}^{2}\right)
\end{aligned}
$$

are $C^{\infty}$-smooth. In addition, we have

$$
\partial_{a} c(a, d)>0
$$

for all $(a, d) \in \mathcal{T}$.

Proof. The $C^{1}$-smoothness of the maps $(c, \Phi)$ follows from [28, Thm. 2.3]. On account of the smoothness of the function $g$, this can readily be extended to the desired $C^{\infty}$-smoothness by using the ideas in [28, section 8] to set up an implicit function argument along the lines of [33, Prop. 6.5].

Differentiating (4.3) with respect to $a$, we compute

$$
-\left[\partial_{a} c\right] \Phi^{\prime}-c\left[\partial_{a} \Phi^{\prime}\right]=d \mathcal{J} \Delta^{+}\left[\partial_{a} \Phi\right]+D G(\Phi ; a, d) \partial_{a} \Phi+\partial_{a} G(\Phi ; a, d),
$$

which gives

$$
-\left[\partial_{a} c\right] \Phi^{\prime}+\mathcal{L}_{a, d} \partial_{a} \Phi=\partial_{a} G(\Phi ; a, d) .
$$

Applying (4.12) and noting that $\partial_{a} g(u ; a)=-u(1-u)<0$ for all $u \in(0,1)$, we obtain

$$
-\partial_{a} c=\int_{-\infty}^{\infty}\left\langle\partial_{a} G(\Phi(\xi) ; a, d), \Psi(\xi)\right\rangle d \xi<0,
$$

as desired.

Corollary 4.4. If $(a, d) \in \mathcal{T}$, then also $\left(a^{\prime}, d\right) \in \mathcal{T}$ for all $\left(a^{\prime}, d\right) \in \Omega_{-}$with $a^{\prime} \geq a$.

Proof of Theorem 2.2. The statements follow directly from Lemmas 4.1-4.3.

4.2. Characterization of waves. We now set out to derive conditions that guarantee either $c(a, d)=0$ or $c(a, d)>0$. To this end, we introduce the functions

$$
u_{a, d}(v)=v-\frac{g(v ; a)}{2 d}, \quad v_{a, d}(u)=u-\frac{g(u ; a)}{2 d}
$$

and note that

$$
\bar{u}_{\#}(a, d)=u_{a, d}\left(\bar{v}_{\#}(a, d)\right), \quad \bar{v}_{\#}(a, d)=v_{a, d}\left(\bar{u}_{\#}(a, d)\right)
$$

for each $\# \in\{A, B, C\}$ and $(a, d) \in \Omega_{-}$. The local extrema of these functions are located at the critical points

$$
\begin{aligned}
\gamma_{c ; \pm}(a, d) & =\frac{1}{3}(a+1) \pm \frac{1}{3} \sqrt{a^{2}-a+1-6 d} \\
& =u_{\mathrm{infl}}(a) \pm \sqrt{\frac{1}{3}\left(g^{\prime}\left(u_{\mathrm{infl}}(a) ; a\right)-2 d\right)} .
\end{aligned}
$$

One can verify that the functions $u_{a, d}$ and $v_{a, d}$ are strictly decreasing on $\left[\gamma_{c ;-}(a, d), \gamma_{c ;+}(a, d)\right]$ and strictly increasing outside this interval. The following ordering result exploits this characterization and will allow us to establish $c(a, d)=0$ for a significant portion of the parameter set $\Omega_{-}$. 
Lemma 4.5. For any $(a, d) \in \bar{\Omega}_{-}$we have the ordering

$$
0 \leq \gamma_{c ;-}(a, d) \leq a \leq \gamma_{c ;+}(a, d) \leq \bar{v}_{B}(a, d)
$$

If $(a, d) \in \Omega_{-}$, then the inequalities in (4.21) are all strict.

Proof. Let us first fix $(a, d) \in \Omega_{-}$. We note that $2 d<2 d_{+}(a)=\frac{g^{\prime}(a ; a)}{2} \leq \frac{g^{\prime}\left(u_{\text {inf }}(a) ; a\right)}{2}$, which implies that $\gamma_{c ; \pm}(a, d)$ are well defined. In addition, this allows us to compute

$$
u_{a, d}^{\prime}(a)=1-\frac{g^{\prime}(a ; a)}{2 d} \leq-1,
$$

which means that $a \in\left(\gamma_{c ;-}(a, d), \gamma_{c ;+}(a, d)\right)$.

In particular, the function $v \mapsto u_{a, d}(v)$ is strictly decreasing on $\left[a, \gamma_{c ;+}(a, d)\right]$. On account of the orderings

$$
u_{a, d}\left(\bar{v}_{A}(a, d)\right)=\bar{u}_{A}(a, d)<\bar{u}_{B}(a, d)=u_{a, d}\left(\bar{v}_{B}(a, d)\right), \quad a<\bar{v}_{A}(a, d)<\bar{v}_{B}(a, d),
$$

we hence cannot have $\bar{v}_{B}(a, d) \leq \gamma_{c ;+}(a)$. The results for the general case $(a, d) \in \bar{\Omega}_{-}$now follow by continuity.

Lemma 4.6. Consider any $(a, d) \in \Omega_{-}$for which $d \leq \frac{1}{8}(1-a)^{2}$. Then we have $c(a, d)=0$.

Proof. Fix any $0<a<1$, write $d_{*}=\frac{1}{8}(1-a)^{2}$, and suppose that $\left(a, d_{*}\right) \in \Omega_{-}$. On account of Corollary 4.4 it suffices to show that $c\left(a, d_{*}\right)=0$. Assuming to the contrary that $c=c\left(a, d_{*}\right)>0$, we compute

$$
\begin{aligned}
0 & >-c \Phi_{v}^{\prime}(\xi) \\
& =d_{*}\left[\Phi_{u}(\xi+1)+\Phi_{u}(\xi-1)-2 \Phi_{v}(\xi)\right]+g\left(\Phi_{v}(\xi) ; a\right) \\
& >-2 d_{*} \Phi_{v}(\xi)+g\left(\Phi_{v}(\xi) ; a\right) \\
& =-2 d_{*} u_{a, d_{*}}\left(\Phi_{v}(\xi)\right) .
\end{aligned}
$$

Since $0<\gamma_{c ;+}\left(a, d_{*}\right)<\bar{v}_{B}\left(a, d_{*}\right)$, there exists $\xi_{*} \in \mathbb{R}$ for which $\Phi_{v}\left(\xi_{*}\right)=\gamma_{c ;+}\left(a, d_{*}\right)$. The key point is that

$$
u_{a, d_{*}}\left(\gamma_{c ;+}\left(a, d_{*}\right)\right)=0
$$

which allows us to obtain a contradiction by picking $\xi=\xi_{*}$ in (4.24).

We now set out to derive a (nonsharp) condition that guarantees $c(a, d)>0$. The strategy will be to rule out the existence of standing bichromatic waves. Let us therefore pick any $(a, d) \in \Omega_{-} \backslash \mathcal{T}$, which implies $c(a, d)=0$. Writing $\left(\Phi_{u}, \Phi_{v}\right)=\Phi(a, d)$ for the corresponding profile, we introduce the sequence

$$
\left(u_{i}, v_{i}\right)=\left(\Phi_{u}(2 i), \Phi_{v}(2 i+1)\right),
$$

which satisfies the limits

$$
\lim _{i \rightarrow-\infty}\left(u_{i}, v_{i}\right)=(0,0), \quad \lim _{i \rightarrow+\infty}\left(u_{i}, v_{i}\right)=\left(\bar{u}_{B}(a, d), \bar{v}_{B}(a, d)\right),
$$




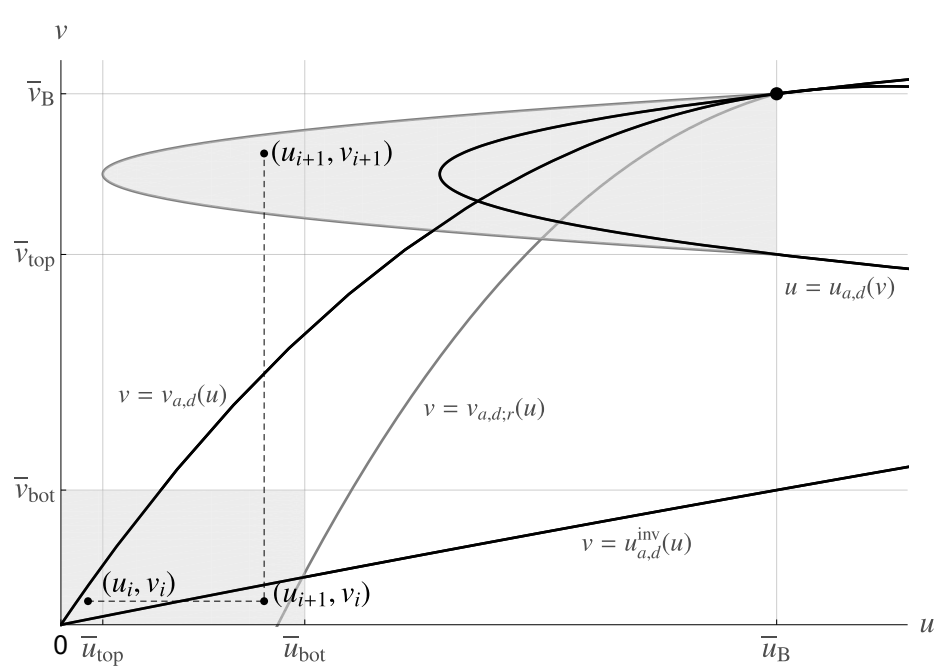

Figure 9. Illustration of the two reflections described by the system (4.30). The values have been modified for illustrative purposes, since the real regions are minuscule.

together with the difference equation

$$
\begin{aligned}
& 0=d\left[v_{i}+v_{i-1}-2 u_{i}\right]+g\left(u_{i} ; a\right), \\
& 0=d\left[u_{i+1}+u_{i}-2 v_{i}\right]+g\left(v_{i} ; a\right) .
\end{aligned}
$$

Applying a shift to the first equation, we obtain the implicit system

$$
\begin{aligned}
& v_{i+1}=2\left[u_{i+1}-\frac{g\left(u_{i+1} ; a\right)}{2 d}\right]-v_{i}, \\
& u_{i+1}=2\left[v_{i}-\frac{g\left(v_{i} ; a\right)}{2 d}\right]-u_{i},
\end{aligned}
$$

which can be written as

$$
\begin{aligned}
& v_{i+1}=2 v_{a, d}\left(u_{i+1}\right)-v_{i}, \\
& u_{i+1}=2 u_{a, d}\left(v_{i}\right)-u_{i} .
\end{aligned}
$$

In particular, we can obtain $\left(u_{i+1}, v_{i+1}\right)$ by first reflecting $\left(u_{i}, v_{i}\right)$ horizontally through the curve $u=u_{a, d}(v)$ and then vertically through the curve $v=v_{a, d}(u)$. Based on this geometric intuition, we set out to construct a rectangle

$$
\left[\bar{u}_{\mathrm{top}}(a, d), \bar{u}_{B}(a, d)\right] \times\left[\bar{v}_{\mathrm{top}}(a, d), \bar{v}_{B}(a, d)\right]
$$

that must contain $\left(u_{i_{0}+1}, v_{i_{0}+1}\right)$ for some critical $i_{0}$, together with a rectangle

$$
\left[0, \bar{u}_{\text {bot }}(a, d)\right] \times\left[0, \bar{v}_{\text {bot }}(a, d)\right]
$$

that must contain the intermediate point $\left(u_{i_{0}+1}, v_{i_{0}}\right) ;$ see Figure 9. 
Lemma 4.7. There exist continuous functions

$$
\left(\bar{v}_{\text {bot }}, \bar{v}_{\text {top }}\right): \Omega_{-} \rightarrow[0,1]^{2}
$$

that satisfy the inequalities

$$
0<\bar{v}_{\text {bot }}(a, d)<\gamma_{c ;-}(a, d)<\bar{v}_{\text {top }}(a, d)<\gamma_{c ;+}(a, d)<\bar{v}_{B}(a, d)
$$

together with the identities

$$
u_{a, d}\left(\bar{v}_{\mathrm{bot}}(a, d)\right)=u_{a, d}\left(\bar{v}_{\mathrm{top}}(a, d)\right)=\bar{u}_{B}(a, d)
$$

for each $(a, d) \in \Omega_{-}$. Furthermore, these functions can be continuously extended to $\bar{\Omega}_{-}$in such a way that (4.35) holds whenever $d>0$.

Proof. Pick any $(a, d) \in \Omega_{-}$. On account of the identity $u_{a, d}(0)=0$ and the inequalities

$$
u_{a, d}\left(\gamma_{c ;+}(a, d)\right)<u_{a, d}\left(\bar{v}_{B}(a, d)\right)=\bar{u}_{B}(a, d)<a=u_{a, d}(a)
$$

the equation $u_{a, d}(v)=\bar{u}_{B}(a, d)$ has three distinct solutions on $\left(0, \bar{v}_{B}(a, d)\right]$. Using the fact that $\bar{u}_{B}(a, 0)=0$, these solutions can be extended continuously to $d=0$ by writing $\bar{v}_{\text {bot }}(a, 0)=0$ and $\bar{v}_{\text {top }}(a, 0)=a$. The extension to $d=d_{-}(a)>0$ can be achieved by standard continuity arguments.

For any $(a, d) \in \bar{\Omega}_{-}$with $d>0$ we now define the constant

$$
\bar{u}_{\text {top }}(a, d)=2 u_{a, d}\left(\gamma_{c ;+}(a, d)\right)-\bar{u}_{B}(a, d) .
$$

We note that $\left(\bar{u}_{\text {top }}(a, d), \gamma_{c ;+}(a, d)\right)$ can be seen as the horizontal reflection of $\left(\bar{u}_{B}(a, d), \gamma_{c ;+}(a, d)\right)$ through the curve $u=u_{a, d}(v)$.

Lemma 4.8. Pick any $(a, d) \in \Omega_{-}$for which $c(a, d)=0$, and consider the sequence $\left\{\left(u_{i}, v_{i}\right)\right\}$ defined in (4.26). Then there exists $i_{0} \in \mathbb{Z}$ for which

$$
\left(\bar{u}_{\text {top }}(a, d), \bar{v}_{\text {top }}(a, d)\right) \leq\left(u_{i_{0}+1}, v_{i_{0}+1}\right) \leq\left(\bar{u}_{B}(a, d), \bar{v}_{B}(a, d)\right),
$$

while

$$
(0,0) \leq\left(u_{i_{0}}, v_{i_{0}}\right) \leq\left(\bar{u}_{B}(a, d), \bar{v}_{\text {bot }}(a, d)\right) .
$$

Proof. For any $i \in \mathbb{Z}$ we have the inequalities

$$
u_{i} \leq u_{i+1} \leq \bar{u}_{B}(a, d),
$$

which implies that we must have $u_{i} \leq u_{a, d}\left(v_{i}\right) \leq u_{i+1}$. In particular, we see that

$$
u_{a, d}\left(v_{i}\right) \leq \bar{u}_{B}(a, d)
$$

which implies that

$$
v_{i} \notin\left(\bar{v}_{\mathrm{bot}}(a, d), \bar{v}_{\mathrm{top}}(a, d)\right) .
$$


In addition, we have

$$
u_{i}=2 u_{a, d}\left(v_{i}\right)-u_{i+1} \geq 2 u_{a, d}\left(v_{i}\right)-\bar{u}_{B}(a, d) .
$$

In particular, for every $i$ we have either

$$
(0,0) \leq\left(u_{i}, v_{i}\right) \leq\left(\bar{u}_{B}(a, d), \bar{v}_{\text {bot }}(a, d)\right)
$$

or

$$
\left(\bar{u}_{\text {top }}(a, d), \bar{v}_{\text {top }}(a, d)\right) \leq\left(u_{i}, v_{i}\right) \leq\left(\bar{u}_{B}(a, d), \bar{v}_{B}(a, d)\right) .
$$

The limits (4.27) imply that there exists $M \gg 1$ so that (4.44) holds for all $i \leq-M$ and (4.45) holds for all $i \geq M$. In particular, there must be a jump between these two sets.

For any $(a, d) \in \bar{\Omega}_{-}$with $d>0$, the inequalities (4.34) imply that the function $u_{a, d}$ is strictly increasing on $\left[0, \bar{v}_{\text {bot }}(a, d)\right]$. This allows us to define an inverse

$$
u_{a, d}^{\text {inv }}:\left[0, \bar{u}_{B}(a, d)\right] \rightarrow\left[0, \bar{v}_{\text {bot }}(a, d)\right] .
$$

In addition, we introduce the function

$$
v_{a, d ; r}(u)=2 v_{a, d}(u)-\bar{v}_{B}(a, d),
$$

which can be interpreted as the vertical reflection of the line $v=\bar{v}_{B}(a, d)$ through the curve $v=v_{a, d}(u)$.

For any $(a, d) \in \bar{\Omega}_{-}$with $d>0$, these definitions allow us to introduce the notation

$$
\bar{u}_{\text {bot }}(a, d)=\max \left\{u \in\left[0, \bar{u}_{B}(a, d)\right]: v_{a, d ; r}(u)=u_{a, d}^{\mathrm{inv}}(u)\right\} .
$$

On account of the inequalities

$$
v_{a, d ; r}(0)<0=u_{a, d}^{\mathrm{inv}}(0)<u_{a, d}^{\mathrm{inv}}\left(\bar{u}_{B}(a, d)\right)=\bar{v}_{\mathrm{bot}}(a, d)<\bar{v}_{B}(a, d)=v_{a, d ; r}\left(\bar{u}_{B}(a, d)\right),
$$

one can verify that $\bar{u}_{\text {bot }}$ is well defined and continuous in $(a, d)$.

Lemma 4.9. Pick $(a, d) \in \Omega_{-}$, and assume that

$$
\bar{u}_{\text {bot }}(a, d)<\bar{u}_{\text {top }}(a, d)
$$

holds. Then we have $c(a, d)>0$.

Proof. Suppose to the contrary that $c(a, d)=0$, and consider the sequence $\left\{\left(u_{i}, v_{i}\right)\right\}$ defined by (4.26), together with the critical value $i_{0} \in \mathbb{Z}$ that appears in Lemma 4.8. Since the sequence $\left\{u_{i}\right\}$ is nondecreasing, we have $u_{i_{0}+1} \geq u_{i_{0}}$, which implies that

$$
u_{i_{0}+1} \geq u_{a, d}\left(v_{i_{0}}\right) .
$$

Exploiting $0 \leq v_{i_{0}} \leq \bar{v}_{\text {bot }}(a, d)$ this gives

$$
0 \leq v_{i_{0}} \leq u_{a, d}^{\mathrm{inv}}\left(u_{i_{0}+1}\right) .
$$

On the other hand, the inequality $v_{i_{0}+1} \leq \bar{v}_{B}(a, d)$ yields

$$
v_{i_{0}} \geq v_{a, d ; r}\left(u_{i_{0}+1}\right) \text {. }
$$

Combining this with (4.52), we see that $u_{i_{0}+1} \leq \bar{u}_{\text {bot }}(a, d)$. However, (4.38) implies that $u_{i_{0}+1} \geq \bar{u}_{\text {top }}(a, d)$, which yields the desired contradiction. 


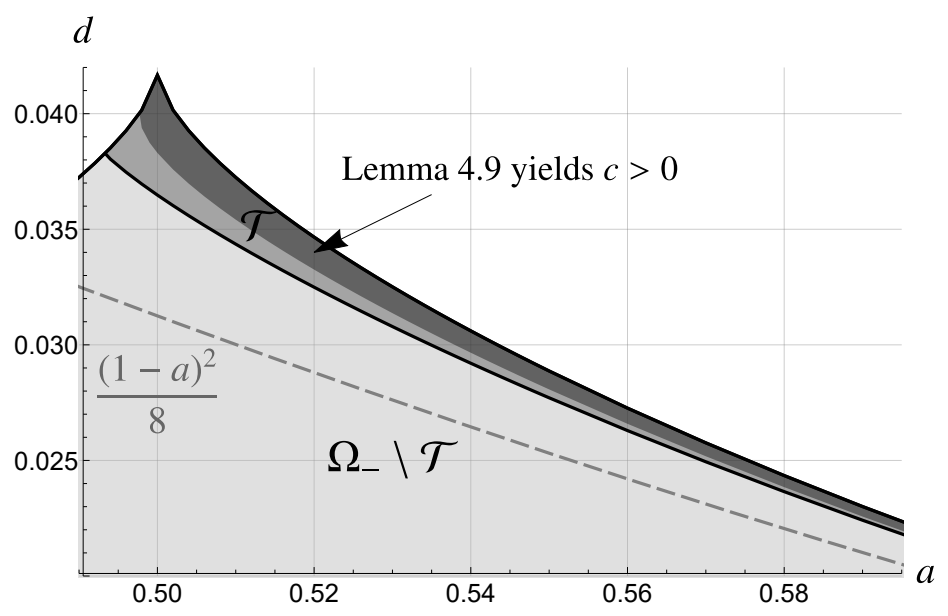

Figure 10. The darkest region contains all pairs $(a, d)$ where the assumption $\bar{u}_{\mathrm{bot}}(a, d)<\bar{u}_{\mathrm{top}}(a, d)$ was verified numerically. We also include the boundaries of the sets $\mathcal{T}$ and $\Omega_{-}$as computed numerically by the procedure described in section 2.

4.3. Verification of $\overline{\boldsymbol{u}}_{\text {top }}>\overline{\boldsymbol{u}}_{\text {bot. }}$. In Figure 10 we show where one may numerically verify that the scalar inequality (4.50) holds, which ensures that $c(a, d)>0$. We also plot the curve $d=\frac{(1-a)^{2}}{8}$, below which we have established that $c(a, d)=0$. Taken together, we feel that these results cover a reasonable portion of the parameter space $\Omega_{-}$.

Our final task is to analytically verify (without resorting to any numerics) that $c(a, d)>0$ near the cusp $(a, d)=\left(\frac{1}{2}, \frac{1}{24}\right)$ and the corner $(a, d)=(1,0)$. As preparation, we construct a simplified but weaker version of the condition $\bar{u}_{\text {top }}>\bar{u}_{\text {bot }}$ by exploiting the monotonicity of $v_{a, d ; r}$.

Lemma 4.10. Pick $(a, d) \in \bar{\Omega}_{-}$with $d>0$. Then the function $v_{a, d ; r}$ is strictly increasing on $\left[0, \bar{u}_{B}(a, d)\right]$.

Proof. We note first that $v_{a, d}^{\prime}(u)=1-\frac{g^{\prime}(u ; a)}{2 d}>0$ for $u \in\left[0, u_{\min }(a)\right]$. In particular, we only have to consider the case $\bar{u}_{B}(a, d)>u_{\min }(a)$, which cannot occur for $a=\frac{1}{2}$.

Let us first assume that $0<a<\frac{1}{2}$. Using (3.108) or (3.109), we may conclude that

$$
v_{a, d}^{\prime}\left(\bar{u}_{B}(a, d)\right) \geq v_{+}^{\prime}\left(\bar{u}_{B}(a, d)\right)>0 .
$$

Since $\bar{u}_{B}(a, d) \leq a \leq \gamma_{c ;+}(a, d)$, this implies that $\bar{u}_{B}(a, d) \leq \gamma_{c ;-}(a, d)$. In particular, $v_{a, d}$ and hence $v_{a, d ; r}$ are strictly increasing on $\left[0, \bar{u}_{B}(a, d)\right]$.

It remains to consider $a \in\left(\frac{1}{2}, 1\right)$. Since $\bar{v}_{B}(1-a, d)>u_{\max }(1-a)$, we may use Corollary 3.4 to conclude

$$
\bar{u}_{B}(a, d)=1-\bar{v}_{B}(1-a, d)<1-u_{\max }(1-a)=u_{\min }(a) .
$$

Corollary 4.11. Consider any $(a, d) \in \bar{\Omega}_{-}$with $d>0$, and suppose that

$$
v_{a, d ; r}\left(\bar{u}_{\text {top }}(a, d)\right)>\bar{v}_{\text {bot }}(a, d) .
$$

Then we have $\bar{u}_{\mathrm{top}}(a, d)>\bar{u}_{\mathrm{bot}}(a, d)$. 
Proof. This follows from the uniform bound $u_{a, d}^{\text {inv }} \leq \bar{v}_{\text {bot }}(a, d)$ and the fact that $v_{a, d ; r}$ is strictly increasing.

We now set out to verify the explicit condition (4.56) for the boundary points $\left(a, d_{-}(a)\right)$ with $a \sim 1$ and $a=\frac{1}{2}$. Using the continuity of $\bar{u}_{\text {top }}$ and $\bar{u}_{\text {bot }}$, this means that $c(a, d)>0$ for all $(a, d) \in \Omega_{-}$that are sufficiently close to these critical boundary points.

Lemma 4.12. We have the expansions

$$
\begin{aligned}
& \bar{u}_{B}\left(a, d_{-}(a)\right)=\frac{1}{4}(1-a)^{2}+\frac{1}{8}(1-a)^{3}+O\left((1-a)^{4}\right), \\
& \bar{v}_{B}\left(a, d_{-}(a)\right)=1-\frac{1}{2}(1-a)+O\left((1-a)^{4}\right)
\end{aligned}
$$

as $a \uparrow 1$.

Proof. Exploiting Corollary 3.4 together with the symmetry $d(a)=d(1-a)$, we obtain

$$
\bar{u}_{B}\left(a, d_{-}(a)\right)=1-\bar{v}_{B}\left(1-a, d_{-}(1-a)\right), \quad \bar{v}_{B}\left(a, d_{-}(a)\right)=1-\bar{u}_{B}\left(1-a, d_{-}(1-a)\right) .
$$

The desired expansions hence follow from Proposition SM1.1 in the supplementary material file M118922_01.pdf [local/web 255KB].

Lemma 4.13. We have the expansions

$$
\begin{array}{ll}
\bar{u}_{\text {top }}\left(a, d_{-}(a)\right) & =\frac{1}{4}(a-1)^{2}+O\left((1-a)^{3}\right), \\
v_{a, d_{-}(a) ; r}\left(\bar{u}_{\text {top }}\left(a, d_{-}(a)\right)\right) & =1+O(1-a)
\end{array}
$$

as $a \uparrow 1$.

Proof. These expansions can be found by substitution of (4.57) into the definitions (4.37) and (4.47).

On account of the identity $\gamma_{c ;-}(1,0)=\frac{1}{3}$ and the inequality $\bar{v}_{\text {bot }}(a, d) \leq \gamma_{c ;-}(a, d)$, we see that Corollary 4.11 implies that

$$
\bar{u}_{\text {top }}\left(a, d_{-}(a)\right)>\bar{u}_{\text {bot }}\left(a, d_{-}(a)\right)
$$

whenever $1-a>0$ is sufficiently small.

Lemma 4.14. The inequality (4.56) holds for $(a, d)=\left(\frac{1}{2}, \frac{1}{24}\right)$.

Proof. Writing $\left(a_{\mathrm{cp}}, d_{\mathrm{cp}}\right)=\left(\frac{1}{2}, \frac{1}{24}\right)$, we can explicitly compute

$$
\gamma_{c ;+}\left(a_{\mathrm{cp}}, d_{\mathrm{cp}}\right)=\frac{1}{2}+\frac{1}{6} \sqrt{2},
$$

which, together with the expressions (3.26) and (4.37), yields

$$
\bar{u}_{\text {top }}\left(a_{\mathrm{cp}}, d_{\mathrm{cp}}\right)=\frac{1}{2}-\frac{4}{9} \sqrt{2}+\frac{1}{6} \sqrt{3} .
$$

Using (4.47), we obtain

$$
v_{a_{\mathrm{cp}}, d_{\mathrm{cp}} ; r}\left(\bar{u}_{\mathrm{top}}\left(a_{\mathrm{cp}}, d_{\mathrm{cp}}\right)\right)=\frac{1}{2}-\frac{1240}{243} \sqrt{2}+\frac{229}{54} \sqrt{3} \sim 0.6286 .
$$


In particular, we have

$$
v_{a_{\mathrm{cp}}, d_{\mathrm{cp}} ; r}\left(\bar{u}_{\mathrm{top}}\left(a_{\mathrm{cp}}, d_{\mathrm{cp}}\right)\right)>a_{\mathrm{cp}} \geq \gamma_{c ;-}\left(a_{\mathrm{cp}}, d_{\mathrm{cp}}\right) \geq \bar{v}_{\mathrm{bot}}\left(a_{\mathrm{cp}}, d_{\mathrm{cp}}\right),
$$

as desired.

Proof of Theorem 2.3. Items (i), (ii), and (iii) follow from Lemma 4.6, Corollary 4.4, and Lemma 4.14, respectively. Item (iv) follows from Lemma 4.5, together with Lemma 4.9 and the continuity of the functions $\bar{u}_{\text {bot }}$ and $\bar{u}_{\text {top }}$. Indeed, the expression (2.26) can be rewritten as

$$
\Gamma(a)=\bar{u}_{\text {top }}\left(a, d_{-}(a)\right)-\bar{u}_{\text {bot }}\left(a, d_{-}(a)\right) .
$$

Finally, item (v) follows directly from (4.60).

Acknowledgment. The authors are grateful to the two anonymous referees for valuable suggestions.

\section{REFERENCES}

[1] D. G. Aronson And H. F. Weinberger, Nonlinear diffusion in population genetics, combustion, and nerve pulse propagation, in Partial Differential Equations and Related Topics, Springer, New York, 1975 , pp. 5-49.

[2] P. W. Bates And A. Chmaj, A discrete convolution model for phase transitions, Arch. Ration. Mech. Anal., 150 (1999), pp. 281-305.

[3] J. Bell AND C. Cosner, Threshold behavior and propagation for nonlinear differential-difference systems motivated by modeling myelinated axons, Quart. Appl. Math., 42 (1984), pp. 1-14.

[4] K. Bhattacharya, Microstructure of Martensite: Why It Forms and How It Gives Rise to the ShapeMemory Effect, Oxford Series on Materials Modelling, Oxford University Press, Oxford, UK, 2004.

[5] M. Brucal-Hallare And E. S. Van Vleck, Traveling wavefronts in an antidiffusion lattice Nagumo model, SIAM J. Appl. Dyn. Syst., 10 (2011), pp. 921-959,https://doi.org/10.1137/100819461.

[6] J. W. CAHn, Theory of crystal growth and interface motion in crystalline materials, Acta Met., 8 (1960), pp. $554-562$.

[7] J. W. Cahn, J. Mallet-Paret, And E. S. VAn Vleck, Traveling wave solutions for systems of ODEs on a two-dimensional spatial lattice, SIAM J. Appl. Math., 59 (1998), pp. 455-493, https://doi.org/ $10.1137 /$ S0036139996312703.

[8] J. W. CAHn And A. NoviCK-Cohen, Evolution equations for phase separation and ordering in binary alloys, J. Stat. Phys., 76 (1994), pp. 877-909.

[9] J. W. CAHN AND E. S. VAN VLECK, On the co-existence and stability of trijunctions and quadrijunctions in a simple model, Acta Materialia, 47 (1999), pp. 4627-4639.

[10] V. Celli and N. Flytzanis, Motion of a screw dislocation in a crystal, J. Appl. Phys., 41 (1970), pp. 4443-4447.

[11] X. Chen, J. S. Guo, And C. C. Wu, Traveling waves in discrete periodic media for bistable dynamics, Arch. Ration. Mech. Anal., 189 (2008), pp. 189-236.

[12] L. O. ChuA And L. YAng, Cellular neural networks: Applications, IEEE Trans. Circuits Syst., 35 (1988), pp. 1273-1290.

[13] L. O. Chua And L. YAng, Cellular neural networks: Theory, IEEE Trans. Circuits Syst., 35 (1988), pp. 1257-1272.

[14] S. V. Dmitriev, K. Abe, And T. Shigenari, Domain wall solutions for EHM model of crystal: Structures with period multiple of four, Phys. D, 147 (2000), pp. 122-134.

[15] C. E. Elmer, Finding stationary fronts for a discrete Nagumo and wave equation; construction, Phys. D, 218 (2006), pp. 11-23. 
[16] C. E. Elmer and E. S. VAn Vleck, A variant of Newton's method for the computation of traveling waves of bistable differential-difference equations, J. Dynam. Differential Equations, 14 (2002), pp. 493-517.

[17] C. E. Elmer and E. S. VAN VleCK, Spatially discrete FitzHugh-Nagumo equations, SIAM J. Appl. Math., 65 (2005), pp. 1153-1174, https://doi.org/10.1137/S003613990343687X.

[18] L. C. Evans, H. M. Soner, And P. E. Souganidis, Phase transitions and generalized motion by mean curvature, Comm. Pure Appl. Math., 45 (1992), pp. 1097-1123.

[19] T. E. Faver, Nanopteron-Stegoton Traveling Waves in Spring Dimer Fermi-Pasta-Ulam-Tsingou Lattices, preprint, https://arxiv.org/abs/1710.07376, 2017.

[20] T. E. FAVER AND J. D. WRIGHT, Exact diatomic Fermi-Pasta-Ulam-Tsingou solitary waves with optical band ripples at infinity, SIAM J. Math. Anal., 50 (2018), pp. 182-250, https://doi.org/10.1137/ $15 \mathrm{M} 1046836$.

[21] P. C. FifE AND J. B. MCLEOD, The approach of solutions of nonlinear diffusion equations to travelling front solutions, Arch. Ration. Mech. Anal., 65 (1977), pp. 335-361.

[22] M. I. Freidlin, Limit theorems for large deviations and reaction-diffusion equations, Ann. Probab., 13 (1985), pp. 639-675.

[23] J. GÄRTNER AND M. I. Freidlin, On the propagation of concentration waves in periodic and random media, Soviet Math. Dokl., 20 (1979), pp. 1282-1286.

[24] A. Hoffman and J. Mallet-Paret, Universality of crystallographic pinning, J. Dynam. Differential Equations, 22 (2010), pp. 79-119.

[25] A. Hoffman And J. D. Wright, Nanopteron solutions of diatomic Fermi-Pasta-Ulam-Tsingou lattices with small mass-ratio, Phys. D, 358 (2017), pp. 33-59.

[26] H. J. Hupkes, D. Pelinovsky, And B. Sandstede, Propagation failure in the discrete Nagumo equation, Proc. Amer. Math. Soc., 139 (2011), pp. 3537-3551, https://doi.org/10.1090/ S0002-9939-2011-10757-3.

[27] H. J. Hupkes And B. Sandstede, Stability of pulse solutions for the discrete FitzHugh-Nagumo system, Trans. Amer. Math. Soc., 365 (2013), pp. 251-301.

[28] H. J. Hupkes AND E. S. VAn VleCK, Negative diffusion and traveling waves in high dimensional lattice systems, SIAM J. Math. Anal., 45 (2013), pp. 1068-1135, https://doi.org/10.1137/120880628.

[29] H. J. Hupkes and S. M. Verduyn-Lunel, Analysis of Newton's method to compute travelling waves in discrete media, J. Dynam. Differential Equations, 17 (2005), pp. 523-572.

[30] J. P. KeEner, Propagation and its failure in coupled systems of discrete excitable cells, SIAM J. Appl. Math., 47 (1987), pp. 556-572, https://doi.org/10.1137/0147038.

[31] J. MALlet-PARet, Spatial patterns, spatial chaos and traveling waves in lattice differential equations, in Stochastic and Spatial Structures of Dynamical Systems (Amsterdam, 1995), Konink. Nederl. Akad. Wetensch. Verh. Afd. Natuurk. Eerste Reeks 45, North-Holland, Amsterdam, 1996, pp. 105-129.

[32] J. Mallet-PARet, The Fredholm alternative for functional differential equations of mixed type, J. Dynam. Differential Equations, 11 (1999), pp. 1-48.

[33] J. MALlET-PARET, The global structure of traveling waves in spatially discrete dynamical systems, J. Dynam. Differential Equations, 11 (1999), pp. 49-128.

[34] J. Mallet-Paret, Crystallographic Pinning: Direction Dependent Pinning in Lattice Differential Equations, preprint, Lefschetz Center for Dynamical Systems and Center for Control Sciences, Division of Applied Mathematics, Brown University, Providence, RI, 2001.

[35] J. Nagumo, S. Arimoto, and S. Yoshizawa, An active pulse transmission line simulating nerve axon, Proc. IRE, 50 (1962), pp. 2061-2070.

[36] F. Sélley, Á. Besenyei, I. Z. Kiss, And P. L. Simon, Dynamic control of modern, network-based epidemic models, SIAM J. Appl. Dyn. Syst., 14 (2015), pp. 168-187, https://doi.org/10.1137/130947039.

[37] N. Shigesada, K. Kawasaki, and E. Teramoto, Traveling periodic waves in heterogeneous environments, Theor. Popul. Biol., 30 (1986), pp. 143-160.

[38] A. SlavíK, Invariant regions for systems of lattice reaction-diffusion equations, J. Differential Equations, 263 (2017), pp. 7601-7626.

[39] L. I. Slepyan, Models and Phenomena in Fracture Mechanics, Springer, Berlin, 2002.

[40] P. STEHLÍK, Exponential number of stationary solutions for Nagumo equations on graphs, J. Math. Anal. Appl., 455 (2017), pp. 1749-1764. 
[41] A. K. Tagantsev, L. E. Cross, and J. Fousek, Domains in Ferroic Crystals and Thin Films, Springer, New York, 2010

[42] A. Vainchtein And E. S. Van Vleck, Nucleation and propagation of phase mixtures in a bistable chain, Phys. Rev. B, 79 (2009), 144123.

[43] A. Vainchtein, E. S. Van Vleck, and A. Zhang, Propagation of periodic patterns in a discrete system with competing interactions, SIAM J. Appl. Dyn. Syst., 14 (2015), pp. 523-555, https://doi.org/10. 1137/140969348.

[44] H. YaGisita, Backward global solutions characterizing annihilation dynamics of travelling fronts, Publ. Res. Inst. Math. Sci., 39 (2003), pp. 117-164.

[45] B. ZinNer, Existence of traveling wavefront solutions for the discrete Nagumo equation, J. Differential Equations, 96 (1992), pp. 1-27. 